\title{
Some remarks on Lagrangian and Poisson reduction for field theories
}

\author{
Marco Castrillón López ${ }^{\mathrm{a}, *}$, Jerrold E. Marsden ${ }^{\mathrm{b}}$ \\ a Dept. Geometría y Topología, Facultad de CC. Matemáticas, Universidad Complutense de Madrid, \\ Madrid 28040, Spain \\ ${ }^{\mathrm{b}}$ Control and Dynamical Systems 107-81, California Institute of Technology, Pasadena, CA 91125, USA
}

Received 17 December 2002; accepted 28 January 2003

\begin{abstract}
Given a Hamiltonian system on a fiber bundle, the Poisson covariant formulation of the Hamilton equations is described. When the fiber bundle is a $G$-principal bundle and the Hamiltonian density is $G$-invariant, the reduction of this formulation is studied thus obtaining the analog of the Lie-Poisson reduction for field theories. The relation of this reduction with the Lagrangian reduction and the Lagrangian and Poisson reduction for electromagnetism are also analyzed.

(c) 2003 Elsevier Science B.V. All rights reserved.
\end{abstract}

MSC: 70 S05

Subj. Clss.: Classical field theory

Keywords: Lagrangian and Poisson reduction; Field theories

\section{Introduction}

There is a long history of reduction theory for symplectic and Poisson manifolds, as described in, for instance, [26]. If one takes the point of view of infinite dimensional fields, then this same formalism of symplectic and Poisson reduction can, and has been applied quite successfully, as in, for instance, to fluids in [25] (inspired by the work of Arnold and others), to electromagnetism and plasma physics in [24] (inspired by work of Morrison and others) and to Yang-Mills type equations in [16,29].

It is important to keep in mind that there is a Lagrangian reduction counterpart to the symplectic and Poisson reduction approach that has also been applied to many field theories.

\footnotetext{
* Corresponding author.

E-mail address: marco_castrillon@mat.ucm.es (J.E. Marsden). 
In Lagrangian reduction one drops variational principles to quotient spaces rather than symplectic or Poisson structures. The most basic of these is the well-known Euler-Poincaré reduction theory as described in [21] for instance, but there have been important extensions of this methodology, inspired by the original work of Marsden and Scheurle [22]. We mention the papers of Cendra et al. [5,17] as typical of these.

However, it has long been recognized that it is quite important to develop a reduction theory that parallels this using the local view of classical fields, as in the classical Poincaré-Cartan and de Donder points of view. The purpose of this paper is to contribute to this effort mainly using the methodology of Poisson reduction.

In terms of what has been done in this direction, there are the results of Castrillón López et al. $[2,4]$ that study covariant Lagrangian reduction for principal bundles. This basically gives a covariant view of the Euler-Poincaré theory. Also, the paper of Fernández et al. [8] (and references therein) gives a useful framework for studying the Lagrangian reduction point of view in field theory.

In our work on Poisson reduction for field theories, we are motivated of course by the Poisson reduction theory from the infinite dimensional point of view, as described above, as well as some of the intriguing remarks and examples in [19]. For the work on Lagrangian reduction we are motivated by extending the covariant Euler-Poincaré theory to a case where the gauge group action involves the derivative of the group elements. This is the case in electromagnetism and it is what make that case of particular interest.

Our specific accomplishments are as follows:

1. In Sections 2-4 we introduce the basic ingredients of the Hamilton-Cartan theory as well as the covariant Poisson bracket. We refer the reader to the existing literature for many of the results concerning this theory.

2. In Section 5, we develop the theory of Poisson brackets in the context of multisymplectic and polysymplectic manifolds and give the equivalence of the Poisson equations and the Hamilton-Cartan equations.

3. We develop a procedure in Section 6 for dropping the Poisson bracket when the phase bundle is a $G$-principal bundle and the considered symmetry group is the structure group itself. This leads to a covariant theory of Lie-Poisson which, under the appropriate hyper-regularity conditions, is equivalent to the covariant Euler-Poincaré reduction mentioned above.

4. We also apply this formalism to the particular case of electromagnetism in Section 7, giving a covariant parallel to what is known from the infinite dimensional point of view. Although it is an example of a non-regular theory, we show that the formalism for both covariant Lagrangian reduction as well as covariant Poisson reduction still hold.

Some general notations and conventions that we shall use are:

1. The Einstein summation convention is assumed.

2. The space of sections of a bundle $Y \rightarrow M$ is denoted by $\Gamma(Y)$.

3. The projection map of a bundle $A \rightarrow B$ is denoted by $\pi_{B A}$.

4. Our base manifold $M$ is assumed to be compact (only for technical simplicity). 


\section{Preliminaries}

The jet bundle. We will need to recall a few notations about jet bundles. For a general exposition of jet bundles, we refer the reader to, for example, [13,28] or to [1].

Given a fiber bundle $\pi_{M Y}: Y \rightarrow M$, we say that two local sections $s: U \rightarrow Y$, $s^{\prime}: U^{\prime} \rightarrow Y$ represent the same jet $j_{x}^{1} s$ at $x \in U \cap U^{\prime}$ iff $s(x)=s^{\prime}(x)$ and $T_{x} s=T_{x} s^{\prime}$. In fact, representing the same jet at $x \in M$ is an equivalence relation, the space of classes of which is denoted by $J_{x}^{1} Y$. The total space $J^{1} Y=\bigcup_{x \in M} J_{x}^{1} Y$ can be endowed with a smooth structure such that the target mapping $J^{1} Y \rightarrow Y, j_{x}^{1} s \mapsto s(x)$, and the source mapping $J^{1} Y \rightarrow M, j_{x}^{1} s \mapsto x$, are fibrations. The fibration $J^{1} Y \rightarrow Y$ is an affine bundle modeled over the vector bundle $\pi_{M E}^{*}\left(T^{*} M\right) \otimes V Y$, where $V Y \subset T Y$ is the vertical bundle, that is, the subbundle of $T Y$ tangent to the fibration $\pi_{M Y}$.

Given a fiber coordinate system $\left(x^{i}, y^{a}\right), 1 \leq i \leq n=\operatorname{dim} M, 1 \leq a \leq m$, on $E$ we define the coordinate system $\left(x^{i}, y^{a}, y_{i}^{a}\right)$ on $J^{1} Y$ by the condition

$$
y_{i}^{a}\left(j_{x}^{1} s\right):=\frac{\partial y^{a}(s)}{\partial x^{i}}(x) .
$$

We thus see that $\operatorname{dim} J^{1} Y=n+m+n m$.

The bundle of connections. Recall that an Ehresmann connection on a fiber bundle $Y \rightarrow$ $M$ is a distribution $\mathcal{A}$ of horizontal complements to the vertical subbundle, that is, for every $y \in Y$, we have $T_{y} Y=V_{y} Y \oplus \mathcal{A}_{y}$. As the elements of the jet bundle $J^{1} Y$ can be understood as a horizontal subspace by the relation $j_{x}^{1} s \leftrightarrow \operatorname{Im} T_{X} s$, an Ehresmann connections on $Y$ can be defined as a section of the bundle $J^{1} Y \rightarrow Y$ (see, for example, [20] or [28]). A relevant particular case happens when the $Y \rightarrow M$ is a principal fiber bundle, say $P \rightarrow M$, with structure group $G$. In this case the Ehresmann connections are taken to be $G$-invariant, that is, the distribution $\mathcal{A}$ is invariant under $T R_{g}$ for any $g \in G$, where $R_{g}$ stands for the right action of $G$ on $P$. Hence, principal connections may be regarded as sections of the bundle $\left(J^{1} P\right) / G \rightarrow P / G=M$ obtained by taking of quotient of the jet bundle by $G$. This bundle, called the bundle of connections and denoted by $C \rightarrow M$, is an affine bundle modeled over the vector bundle $T^{*} M \otimes \tilde{\mathfrak{g}} \rightarrow M$, where $\tilde{\mathfrak{g}} \rightarrow M$ is the adjoint bundle. See [3] and references therein for a more detailed study of $C \rightarrow M$.

The Lagrangian formalism. We shall also need to recall some of the basic notations and results from the variational formalism for field theories. A first-order Lagrangian density is a smooth fiber map $\mathcal{L}: J^{1} Y \rightarrow \wedge^{n} T^{*} M$. We say that a (local) section $s$ of the bundle $Y \rightarrow M$ is a critical solution of the variational problem defined by $\mathcal{L}$ if for every smooth family of sections $\left\{s_{\varepsilon}\right\}_{\varepsilon \in \mathbb{R}}$ such that $s_{0}=s$, we have

$$
\left.\frac{\mathrm{d}}{\mathrm{d} \varepsilon}\right|_{\varepsilon=0} \int_{M} \mathcal{L} \circ j^{1} s_{\varepsilon}=0 .
$$

We assume that the manifold $M$ is oriented by a volume form $\mathbf{v}$ and we shall write $\mathcal{L}=L \mathbf{v}$, which defines $L \in C^{\infty}\left(J^{1} Y\right)$. There is an operator $\mathcal{E} \mathcal{L}: \Gamma(Y) \rightarrow \Gamma\left(V^{*} Y\right)$, called the Euler-Lagrange operator, whose local expression is

$$
\mathcal{E} \mathcal{L}(s)=\left(\frac{\partial L}{\partial y^{\alpha}} \circ j^{1} s-\frac{\mathrm{d}}{\mathrm{d} x^{i}}\left(\frac{\partial L}{\partial y_{i}^{\alpha}} \circ j^{1} s\right)\right) \otimes \mathrm{d} y^{\alpha}
$$


for a local coordinate system $\left(x^{i}\right)$ on $M$ with $\mathbf{v}=\mathrm{d} x^{1} \wedge \cdots \wedge \mathrm{d} x^{n}$. A standard result in the calculus of variations says that $s$ is critical if and only if $\mathcal{E} \mathcal{L}(s)=0$.

\section{The multisymplectic formalism}

The dual jet bundle. There are many variants of the multisymplectic formalism in the literature and many different notations. Thus, we will need to review our notation and set up. We shall follow the version given in [13], see also [27].

Given a fiber bundle $Y \rightarrow M$, one defines the dual jet bundle $J^{1} Y^{*}$ to be the vector bundle over $Y$ whose fiber at $y \in Y_{x}$ is the set of affine morphisms from $\left(J^{1} Y\right)_{y}$ to the bundle of $n$-forms on $M$, which we denote by $\left(\bigwedge^{n} T^{*} M\right)_{x}$, that is

$$
J^{1} Y^{*}=\operatorname{Aff}\left(J^{1} Y, \bigwedge^{n} T^{*} M\right)
$$

Given a fiber chart $\left(x^{i}, y^{a}\right)$ on $Y$, fiber coordinates $\left(x^{i}, y^{a}, p_{a}^{i}, p\right)$ on $J^{1} Y^{*}$ are defined such that an affine map has the form

$$
y_{i}^{a} \mapsto\left(p+p_{a}^{i} y_{i}^{a}\right) \mathrm{d} x^{1} \wedge \cdots \wedge \mathrm{d} x^{n} .
$$

We thus see that $\operatorname{dim} J^{1} Y^{*}=n+m+n m+1$.

There is another characterization of the space $J^{1} Y^{*}$. Let $Z$ be the subbundle of $\bigwedge^{n} T^{*} Y$ whose fiber at $y \in Y$ is given by

$$
Z_{y}=\left\{\left.z \in\left(\bigwedge^{n} T^{*} Y\right)_{y}\right|_{\mathbf{i}_{u}} \mathbf{i}_{v} z=0 \text { for all } u, v \in V_{y} Y\right\},
$$

that is, $Z$ consists of $n$-covectors annihilated by the contraction (interior product) by two vertical vectors. Locally, elements of $Z$ can be written as

$$
z=p \mathrm{~d}^{n} x+p_{a}^{i} \mathrm{~d} y^{a} \wedge \mathrm{d}^{n-1} x_{i},
$$

where $\mathrm{d}^{n} x=\mathrm{d} x^{1} \wedge \cdots \wedge \mathrm{d} x^{n}$ and $\mathrm{d}^{n-1} x_{i}=i_{\partial / \partial x^{i}} \mathrm{~d}^{n} x$. We define the mapping

$$
\Phi: Z \rightarrow J^{1} Y^{*}
$$

by setting $\Phi(z)\left(j_{x}^{1} s\right)=s^{*} z \in \bigwedge^{n} T^{*} M$, for $z \in Z_{y}, j_{x}^{1} s \in\left(J^{1} Y\right)_{y}$. This mapping is a vector bundle isomorphism whose local expression simply equals the coordinate systems defined on $J^{1} Y^{*}$ and $Z$ above.

This alternative characterization of the dual jet bundle enables one to introduce in a natural way the multisymplectic form on $J^{1} Y^{*}$. We first define the canonical $n$-form $\Theta_{\Lambda}$ on $\bigwedge^{n} T^{*} Y$ by

$$
\Theta_{\Lambda}(z)\left(u_{1}, \ldots, u_{n}\right)=z\left(T \pi_{Y_{\Lambda}} u_{1}, \ldots, T \pi_{Y_{\Lambda}} u_{n}\right),
$$

where $z \in \bigwedge^{n} T^{*} Y, u_{1}, \ldots, u_{n} \in T_{z}\left(\bigwedge^{n} T^{*} Y\right)$ and $\pi_{Y_{\Lambda}}$ is the projection $\bigwedge^{n} T^{*} Y \rightarrow Y$. If $i_{\Lambda Z}: Z \hookrightarrow \bigwedge^{n} T^{*} Y$ denotes the inclusion, we define the canonical $n$-form on $Z$ (resp. on $\left.J^{1} Y^{*}\right)$ to be the pull-back $i_{\Lambda Z}^{*} \Theta_{\Lambda}\left(\operatorname{resp} .\left(\Phi^{-1}\right)^{*} i_{\Lambda Z}^{*} \Theta_{\Lambda}\right)$. For the sake of simplicity, we 
denote the canonical form as $\Theta$ both on $Z$ and on $J^{1} Y^{*}$. The canonical multisymplectic $(n+1)$-form is defined as

$$
\Omega=-\mathrm{d} \Theta \text {. }
$$

One easily finds that the local expressions for $\Theta$ is

$$
\Theta=p_{a}^{i} \mathrm{~d} y^{a} \wedge \mathrm{d}^{n-1} x_{i}+p \mathrm{~d}^{n} x,
$$

and so

$$
\Omega=\mathrm{d} y^{a} \wedge \mathrm{d} p_{a}^{i} \wedge \mathrm{d}^{n-1} x_{i}-\mathrm{d} p \wedge \mathrm{d}^{n} x .
$$

Let $\mathcal{L}: J^{1} Y \rightarrow \bigwedge^{n} T^{*} M$ be a Lagrangian density. The covariant Legendre transformation is the fiber map

$$
\mathbb{F} \mathcal{L}: J^{1} Y \rightarrow J^{1} Y^{*}
$$

over $Y$ that is defined to be the first-order vertical Taylor approximation to $\mathcal{L}$; that is

$$
\mathbb{F} \mathcal{L}\left(j_{x}^{1} s\right)\left(j_{x}^{1} s^{\prime}\right)=\mathcal{L}\left(j_{x}^{1} s\right)+\left.\frac{\mathrm{d}}{\mathrm{d} \varepsilon}\right|_{\varepsilon=0} \mathcal{L}\left(j_{x}^{1} s+\varepsilon\left(j_{x}^{1} s^{\prime}-j_{x}^{1} s\right)\right) .
$$

Locally, in coordinates, we have

$$
p_{a}^{i}=\frac{\partial L}{\partial y_{i}^{a}}, \quad p=L-\frac{\partial L}{\partial y_{i}^{a}} y_{i}^{a},
$$

where $\mathcal{L}=L \mathrm{~d}^{n} x$.

The following proposition is the basic link between this approach to multisymplectic forms and the classical Poincaré-Cartan formalism in the calculus of variations, as in, for example, $[11,14]$.

Proposition 3.1. Given a Lagrangian density $\mathcal{L}: J^{1} Y \rightarrow \bigwedge^{n} T^{*} M$, the pull-back $\mathbb{F} \mathcal{L}^{*} \Theta$ of the canonical $n$-form $\Theta$ of $J^{1} Y^{*}$ by the Legendre transformation is the classical PoincaréCartan form of $\mathcal{L}$ of the calculus of variations.

Proof. A local computation taking into account Eq. (3.1) and the local expression of the Poincaré-Cartan form (see [10])

$$
\Theta_{\mathcal{L}}=\frac{\partial \mathcal{L}}{\partial y_{i}^{a}} \mathrm{~d} y^{a} \wedge \mathrm{d}^{n-1} x_{i}+\left(L-\frac{\partial}{\partial y_{i}^{a}} y_{i}^{a}\right) \mathrm{d}^{n} x .
$$

The polysymplectic bundle. The polysymplectic formalism provides some interesting alternative perspectives on the geometry of field theories; it goes back to [15]; see also [27].

Since the dimensions of $J^{1} Y$ and $J^{1} Y^{*}$ are different, $\mathbb{F} \mathcal{L}$ can never be a diffeomorphism. This difference is due to the fact that the affine structure of the jet bundle is taken into account in constructing the dual. This can be "fixed" by defining a linear approximation of $\mathcal{L}$ instead of the vertical Taylor approximation. Given a fiber bundle $Y \rightarrow M$ one defines the polysymplectic bundle $\Pi$ over $Y$ as

$$
\pi_{M Y}^{*} T M \otimes_{Y} V^{*} Y \otimes_{Y} \pi_{M Y}^{*}\left(\bigwedge^{n} T^{*} M\right) .
$$


Whenever it is clear that all the bundles are over $Y$, we will simply write

$$
\Pi=T M \otimes V^{*} Y \otimes \bigwedge^{n} T^{*} M
$$

with the pull-back notation omitted. Local coordinates $\left(x^{i}, y^{a}, \pi_{a}^{i}\right)$ are defined by the condition

$$
\xi=\pi_{a}^{i} \frac{\partial}{\partial x^{i}} \otimes \mathrm{d} y^{a} \otimes \mathrm{d}^{n} x
$$

for any $\xi \in \Pi$. Hence $\operatorname{dim} \Pi=n+m+n m$.

Note that the space $\Pi$ can be seen as the space of $\wedge^{n} T^{*} M$-valued forms on $T^{*} M \otimes V Y$, which is precisely the vector bundle over which the affine bundle $J^{1} Y \rightarrow Y$ is modeled. We can thus define the linear Legendre transformation

$$
\widehat{\mathbb{F L}}: J^{1} Y \rightarrow \Pi
$$

to be the fiber map over $Y$ defined as

$$
\widehat{\mathbb{F} \mathcal{L}}\left(j_{x}^{1} s\right)(\varpi)=\left.\frac{\mathrm{d}}{\mathrm{d} \varepsilon}\right|_{\varepsilon=0} \mathcal{L}\left(j_{x}^{1} s+\varepsilon \varpi\right)
$$

for any $\varpi \in T^{*} M \otimes V Y$. Locally we have

$$
\pi_{a}^{i}=\frac{\partial L}{\partial y_{i}^{a}} .
$$

The linear Legendre transformation $\widehat{\mathbb{F} \mathcal{L}}$ can now be a diffeomorphism but one has to pay a price. Even though we can define a canonical vector bundle valued form on $\Pi$ with which the Hamiltonian analysis can be carried out (see, for example, [12]), the manifold $\Pi$ is not endowed with a canonical form as $J^{1} Y^{*}$ is. Nevertheless, the polymomentum bundle and the dual bundle are related as follows.

Proposition 3.2. The fiber map $J^{1} Y^{*} \rightarrow \Pi$ sending $\phi \in \operatorname{Aff}\left(\left(J^{1} Y\right)_{y}, \bigwedge^{n} T_{x}^{*} M\right)=$ $\left(J^{1} Y^{*}\right)_{y}$, to the associated linear morphism $\vec{\phi} \in\left(T_{x} M \otimes V_{y}^{*} Y\right)^{*} \otimes \bigwedge^{n} T_{x}^{*} M=\Pi_{y}$, is a one-dimensional fibration.

Proof. As the map $J^{1} Y^{*} \rightarrow \Pi, \phi \mapsto \vec{\phi}$, is a vector bundle morphism over $Y$, the projection $J^{1} Y^{*} \rightarrow \Pi$ is a fibration whose fibers are the kernel of the morphism. It is evident that the kernel is one-dimensional.

Hamiltonian systems. A Hamiltonian system in the field theoretic context is a pair $(\Pi, \delta)$, where $\delta$ is a section of the bundle $J^{1} Y^{*} \rightarrow \Pi$. Given a Hamiltonian system, the canonical multisymplectic form $\Omega=\mathrm{d} \Theta$ on $J^{1} Y^{*}$ can be pulled back to $\Pi$ thus obtaining an $(n+$ 1)-form $\Omega_{\delta}=\mathrm{d} \Theta_{\delta}$ on $\Pi$. A section $\pi$ of the composite bundle $\Pi \rightarrow Y \rightarrow M$ is said to be a solution of the Hamiltonian system if the following equation holds:

$$
\pi^{*} i_{X} \Omega_{\delta}=\pi^{*} i_{X} \mathrm{~d} \Theta_{\delta}=0
$$


for any vertical vector field $X$ on $\Pi$. We now introduce the Hamiltonian densities. For that we need first the following result (also see [12]).

Proposition 3.3. Given two sections $\delta_{1}$ and $\delta_{2}$ of $J^{1} Y^{*} \rightarrow \Pi$, the $n$-form $\Theta_{\delta_{1}}-\Theta_{\delta_{2}}$ is a horizontal density on $\Pi$, that is, there is a mapping $\mathcal{H}: \Pi \rightarrow \wedge^{n} T^{*} M$ such that $\Theta_{\delta_{1}}-\Theta_{\delta_{2}}=\mathcal{H}$.

Proof. If we locally express a section $\delta$ as

$$
\delta\left(x^{i}, y^{a}, \pi_{a}^{i}\right)=\left(x^{i}, y^{a}, p_{a}^{i}, H_{\delta}\right)
$$

for a certain function $H_{\delta}$, then we have

$$
\Theta_{\delta}=\mathrm{d} y^{a} \wedge \mathrm{d} \pi_{a}^{i} \wedge \mathrm{d}^{n-1} x_{i}-H_{\delta} \wedge \mathrm{d} x^{n},
$$

and the proof immediately follows.

Moreover, an Ehresmann connection $\mathcal{A}: T Y \rightarrow V Y$ on $Y \rightarrow M$ naturally defines a linear section $\delta_{\mathcal{A}}$ of $J^{1} Y^{*} \rightarrow \Pi$ by setting

$$
T_{x} M \otimes V_{p}^{*} P \otimes \bigwedge^{n} T_{x}^{*} M \in w \otimes \xi \otimes \mathbf{v} \mapsto(\xi \circ \mathcal{A}) \wedge i_{w} \mathbf{v} \in Z_{y} \simeq\left(J^{1} Y^{*}\right)_{y} .
$$

Hence, due to Proposition 3.3, given a Hamiltonian system $(\Pi, \delta)$ and a connection $\mathcal{A}$ there exists a density $\mathcal{H}^{\mathcal{A}}$ called the Hamiltonian density defined by $\delta$ and $\mathcal{A}$ such that

$$
\Theta_{\delta}=\Theta_{\delta_{\mathcal{A}}}+\mathcal{H}^{\mathcal{A}}
$$

Hence a section $\pi$ of $\Pi \rightarrow M$ is a solution if and only if

$$
\pi^{*} \mathbf{i}_{X} \mathrm{~d}\left(\Theta_{\delta_{\mathcal{A}}}+\mathcal{H}^{\mathcal{A}}\right)=0 .
$$

Conversely, a Hamiltonian system is thus equally defined by a triplet $(\Pi, \mathcal{A}, \mathcal{H})$, where $\mathcal{A}$ is a connection and $\mathcal{H}$ is a density on $\Pi$, called Hamiltonian density. It is easy to see that the Hamilton equation (3.2) is locally equivalent to

$$
\left(\frac{\partial H}{\partial \pi_{\alpha}^{i}}\right)_{\pi}=\left(\frac{\partial y^{\alpha}}{\partial x^{i}}-\Gamma_{i}^{\alpha}\right)_{\pi}, \quad\left(\frac{\partial H}{\partial y^{\alpha}}\right)_{\pi}=-\left(\frac{\partial \pi_{\alpha}^{i}}{\partial x^{i}}+\frac{\partial \Gamma_{i}^{\beta}}{\partial y^{\alpha}} \pi_{\beta}^{j}\right)_{\pi},
$$

where $\Gamma_{i}^{\alpha}$ stands for the coefficients of the horizontal lift

$$
\frac{\partial}{\partial x^{i}} \mapsto \frac{\partial}{\partial x^{i}}+\Gamma_{i}^{\alpha} \frac{\partial}{\partial y^{\alpha}}
$$

defined by the connection $\mathcal{A}$ and $\mathcal{H}=H \mathrm{~d}^{n} x$. Eq. (3.3) are called the Hamilton-Cartan equations of the Hamiltonian system.

Hyper-regular Lagrangians. Given a Lagrangian density $\mathcal{L}: J^{1} Y \rightarrow \bigwedge^{n} T^{*} M$, we say that $\mathcal{L}$ is hyper-regular if $\mathbb{F} \mathcal{L}$ is a diffeomorphism onto its image, or equivalently, $\widehat{\mathbb{F} \mathcal{L}}$ is a diffeomorphism. Note that $\operatorname{dim} J^{1} P=\operatorname{dim} \Pi=\operatorname{dim} J^{1} P^{*}-1$. 
For a hyper-regular Lagrangian $\mathcal{L}$, we define a section $\delta: \Pi \rightarrow J^{1} P^{*}$ by the equation $\delta=\mathbb{F} \mathcal{L} \circ \widehat{\mathbb{F L}}^{-1}$; that is, we have the commutative diagram

$$
\begin{array}{ccc}
J^{1} Y \quad \stackrel{\mathbb{F} \mathcal{L}}{\rightarrow} & J^{1} Y^{*} \\
\searrow \widehat{\mathbb{F L}} & \downarrow \uparrow \delta \\
& \Pi .
\end{array}
$$

Given a connection $\mathcal{A}$ on $Y \rightarrow M$, the Hamiltonian $\mathcal{H}_{\mathcal{L}}^{\mathcal{A}}$ associated to $\mathcal{L}$ and $\mathcal{A}$ is thus uniquely defined by the condition

$$
\Theta_{\delta}=\Theta_{\delta_{\mathcal{A}}}+\mathcal{H}_{\mathcal{L}}^{\mathcal{A}},
$$

and its local expression reads

$$
\mathcal{H}_{\mathcal{L}}^{\mathcal{A}}=\pi_{\alpha}^{i}\left(\widehat{\mathbb{F L}}^{-1} \circ y_{i}^{\alpha}-\Gamma_{i}^{\alpha}\right) \mathbf{v}-\widehat{\mathbb{F L}}^{-1} \circ \mathcal{L}
$$

The triplet $\left(\Pi, \mathcal{A}, \mathcal{H}_{\mathcal{L}}^{\mathcal{A}}\right)$ is called the Hamiltonian system associated by $\mathcal{L}$ and $\mathcal{A}$.

Remark. There are other alternative (but equivalent) definitions of the Hamiltonian density defined by a Lagrangian density and a connection. For example, it can be understood as translation to $\Pi$ (by means of $\widehat{\mathbb{F} \mathcal{L}}$ ) of the Lagrangian energy $\mathcal{E}_{\mathcal{L}}^{\mathcal{A}}: J^{1} Y \rightarrow \bigwedge^{n} T^{*} M$ defined by $\mathcal{L}$ and $\mathcal{A}$ (see [7]). Another definition can be geometrically done on the subspace $\mathbb{F} \mathcal{L}\left(J^{1} Y\right) \subset J^{1} Y^{*}$ (see [23]). In this case what it is obtained is the density $\delta^{*} \mathcal{H}$.

Theorem 3.4. Let $\mathcal{L}: J^{1} Y \rightarrow \bigwedge^{n} T^{*} M$ be a hyper-regular Lagrangian. Given a section $s$ of $Y \rightarrow M$, we define the section $\pi=\widehat{\mathbb{F L}} \circ j^{1}$ s of the bundle $\Pi \rightarrow M$. Then $s$ is $a$ critical section of the variational problem defined by $\mathcal{L}$ if and only if $\pi$ is a solution of the Hamiltonian system $\left(\Pi, \mathcal{A}, \mathcal{H}_{\mathcal{L}}^{\mathcal{A}}\right)$.

Proof. For a proof, see, for example [23,27].

\section{Poisson forms on $\Pi$}

An $r$-form $F$ on $J^{1} Y^{*}$ is said to be horizontal if $\mathbf{i}_{u} F=0$ for any vertical tangent vector $u$ with respect to the fibration $J^{1} Y \rightarrow M$. In local coordinates we have

$$
F=F_{i_{1} \cdots i_{r}} \mathrm{~d} x^{i_{1}} \wedge \cdots \wedge \mathrm{d} x^{i_{r}}
$$

for the component functions $F_{i_{1} \cdots i_{r}}$ on $J^{1} Y^{*}$. An $s$-multivector field $\chi$ on $J^{1} Y^{*}$ (that is, a section of $\left.\bigwedge^{s} T\left(J^{1} Y^{*}\right) \rightarrow J^{1} Y^{*}\right)$ is said to be vertical if its contraction $\mathbf{i}_{\chi} F$ with any horizontal $s$-form $\chi$ vanishes.

We say that a horizontal $r$-form $F$ on $J^{1} Y^{*}$ is a Poisson form if there is a vertical $(n-r)$-multivector field $\chi_{F}$ on $J^{1} Y^{*}$ such that

$$
\mathbf{i}_{\chi F} \Omega=\mathrm{d} F,
$$


where $\Omega$ is the multisymplectic form on $J^{1} Y^{*}$. Given a horizontal $r$-form $F$ and a horizontal $s$-form $E$ on $J^{1} P^{*}$ we define their Poisson bracket to be

$$
\{F, E\}=(-1)^{r(s-1)} \mathbf{i}_{\chi_{E}} \mathbf{i}_{\chi_{F}} \Omega .
$$

Note that $\{E, F\}$ is an $(r+s+1-n)$-form on $J^{1} Y^{*}$.

Remark. Not every horizontal form is Poisson. In fact we are going to see that this condition is quite restrictive for $(n-1)$-forms. On the other hand, given a Poisson form $F$, the associated multivector field $\chi_{F}$ may not be unique. Actually, $\chi_{E}$ is defined up to an element belonging to $\operatorname{ker} \Omega$, that is a multivector field $\chi$ such that $\mathbf{i}_{\chi} \Omega=0$. In the symplectic setting mechanics, where symplectic form is non-degenerate, we have $\chi=0$, thus having uniqueness, but this is not the case for field theories. In any case, the non-uniqueness of $\chi$ does not obviously give an ambiguous definition of $\{F, E\}$. We explore this issue in the following discussion.

Proposition 4.1. Any function $F: J^{1} P^{*} \rightarrow \mathbb{R}$ is a Poisson 0-form.

Proof. In coordinates

$$
\Omega=\mathrm{d} y^{\alpha} \wedge \mathrm{d} \pi_{\alpha}^{i} \wedge \mathbf{v}_{i}-\mathrm{d} p \wedge \mathbf{v}
$$

where $\mathbf{v}=\mathrm{d} x^{1} \wedge \cdots \wedge \mathrm{d} x^{n}$, and $\mathbf{v}_{i}=i_{\partial / \partial x^{i}} \mathbf{v}$. Given a function $F$ we have

$$
\mathrm{d} F=\frac{\partial F}{\partial x^{i}} \mathrm{~d} x^{i}+\frac{\partial F}{\partial y^{\alpha}} \mathrm{d} y^{\alpha}+\frac{\partial F}{\partial \pi_{\alpha}^{i}} \mathrm{~d} \pi_{\alpha}^{i} .
$$

Hence the multivector field

$$
\chi=\frac{\partial F}{\partial \pi_{\alpha}^{i}} \frac{\partial}{\partial y^{\alpha}} \wedge \mathbf{v}_{i}^{*}-\frac{\partial F}{\partial y^{\alpha}} \frac{\partial}{\partial \pi_{\alpha}^{i}} \wedge \mathbf{v}_{i}^{*}+\frac{\partial F}{\partial x^{i}} \frac{\partial}{\partial p} \mathbf{v}_{i}^{*}
$$

satisfies the condition (4.1), where $\mathbf{v}^{*}=\left(\partial / \partial x^{1}\right) \wedge \cdots \wedge\left(\partial / \partial x^{n}\right)$ and $\mathbf{v}_{i}^{*}=i_{\mathrm{d} x^{i}} \mathbf{v}^{*}$.

Proposition 4.2. If a horizontal $r$-form $F, r>0$, on $J^{1} Y^{*}$ is Poisson, then it is projectable to $\Pi$ (see Proposition 3.2).

Proof. Let

$$
F=F^{i_{1} \cdots i_{s}} \mathbf{v}_{i_{1} \cdots i_{s}}
$$

be the local expression of $F$, with $s=n-r$ and $\mathbf{v}_{i_{1} \cdots i_{s}}=i_{\partial / \partial x^{i_{1}}} \cdots i_{\partial / \partial x^{i_{s}}} \mathbf{v}$. Then

$$
\begin{aligned}
\mathrm{d} F= & \frac{\partial F^{i_{2} \cdots i_{s} j}}{\partial x^{j}} \mathbf{v}_{i_{2} \cdots i_{s}}+\frac{\partial F^{i_{1} \cdots i_{s}}}{\partial y^{\alpha}} \mathrm{d} y^{\alpha} \wedge \mathbf{v}_{i_{1} \cdots i_{s}} \\
& +\frac{\partial F^{i_{1} \cdots i_{s}}}{\partial \pi_{\alpha}^{j}} \mathrm{~d} \pi_{\alpha}^{j} \wedge \mathbf{v}_{i_{1} \cdots i_{s}}+\frac{\partial F^{i_{1} \cdots i_{s}}}{\partial p} \mathrm{~d} p \wedge \mathbf{v}_{i_{1} \cdots i_{s}} .
\end{aligned}
$$


Using the local expression for $\Omega$,

$$
\Omega=\mathrm{d} y^{\alpha} \wedge \mathrm{d} \pi_{\alpha}^{i} \wedge \mathbf{v}_{i}-\mathrm{d} p \wedge \mathbf{v}
$$

for a $s$-multivector $\chi$ to verify that $\mathbf{i}_{\chi} \Omega=\mathrm{d} F$, we see that $\chi$ does not contain any elements $X^{i_{1} \cdots i_{s}}\left(\partial / \partial x^{i_{1}}\right) \wedge \cdots \wedge\left(\partial / \partial x^{i_{s}}\right)$ because they would give terms proportional to $\mathrm{d} y^{\alpha} \wedge \mathrm{d} \pi_{\alpha}^{i} \wedge$ $\mathbf{v}_{i i_{1} \cdots i_{s}}$, which $\mathrm{d} F$ does not contain. But then, the last term of (4.3) cannot exist and hence, the functions $F^{i_{1} \cdots i_{s}}$ do not depend on the variable $p$. Hence $F$ is projectable.

Moreover, if $F$ is a Poisson form, as $\mathbf{i}_{\chi} \Omega=\mathrm{d} F$, from formula (4.2) we easily have that the bracket of two projectable Poisson forms is still a projectable form to $\Pi$. Hence, from now on we will consider that the horizontal Poisson forms are defined on $\Pi$ and will be seen on $J^{1} Y^{*}$ by pulling-back only when needed.

We now study the special case where $F$ is a Poisson $(n-1)$-form. We write

$$
F=F^{i} \mathbf{v}_{i},
$$

and

$$
\mathrm{d} F=\frac{\partial F^{i}}{\partial x^{i}} \mathbf{v}+\frac{\partial F^{i}}{\partial y^{\alpha}} \mathrm{d} y^{\alpha} \wedge \mathbf{v}_{i}+\frac{\partial F^{i}}{\partial \pi_{\alpha}^{j}} \mathrm{~d} \pi_{\alpha}^{j} \wedge \mathbf{v}_{i} .
$$

Let $\chi$ be a vector field on $J^{1} P^{*}$ such that $\mathbf{i}_{\chi} \Omega=\mathrm{d} F$. We write

$$
\chi=X^{\alpha} \frac{\partial}{\partial y^{\alpha}}+X_{\alpha}^{i} \frac{\partial}{\partial \pi_{\alpha}^{i}}+X \frac{\partial}{\partial p} .
$$

Hence, for

$$
\mathbf{i}_{\chi} \Omega=X^{\alpha} \mathrm{d} \pi_{\alpha}^{i} \wedge \mathbf{v}_{i}-X_{\alpha}^{i} \mathrm{~d} y^{\alpha} \wedge \mathbf{v}_{i}-X \mathbf{v}
$$

to be $\mathrm{d} F$, the following conditions must be satisfied:

$$
\frac{\partial F^{i}}{\partial x^{i}}=X, \quad \frac{\partial F^{i}}{\partial y_{\alpha}}=-X_{\alpha}^{i}, \quad \frac{\partial F^{i}}{\partial \pi_{\alpha}^{j}}=\delta_{j}^{i} X^{\alpha} .
$$

These conditions only constraint the behavior of $F$ with respect to $\pi_{\alpha}^{i}$. Actually, we obtain that the general expression of a horizontal $(n-1)$-Poisson form is

$$
F=\left(-\pi_{\alpha}^{i} X^{\alpha}+g^{i}\right) \mathbf{v}_{i}
$$

for any functions $X^{\alpha}=X^{\alpha}(x, y), g^{i}=g^{i}(x, y)$. Moreover, as every closed horizontal form on $\Pi$ is Poisson, we can add a closed term to (4.4). This local expression of the Poisson $(n-1)$-forms can be given in an intrinsic way as follows.

Proposition 4.3. For a vertical vector field $X$ on $Y \rightarrow M$ (locally $\left.X=X^{\alpha} \partial / \partial y^{\alpha}\right)$ we construct a mapping

$$
\Pi=T M \otimes_{Y} V^{*} Y \otimes_{Y} \bigwedge^{n} T^{*} M \rightarrow T M \otimes_{Y} \bigwedge^{n} T^{*} M
$$


by contracting the $V^{*} Y$ part with $X$. By composing this mapping with the natural contraction $T M \otimes_{Y} \bigwedge^{n} T^{*} M \rightarrow \pi_{M Y}^{*}\left(\bigwedge^{n-1} T^{*} M\right)$ we obtain a morphism

$$
\theta_{X}: \Pi \rightarrow \pi_{M Y}^{*}\left(\bigwedge^{n-1} T^{*} M\right)
$$

that is, a horizontal ( $n-1)$-form on $\Pi$. In local coordinates, the result of the composition yields

$$
\theta_{X}\left(\pi_{\alpha}^{i} \frac{\partial}{\partial x^{i}} \otimes \mathrm{d} y^{\alpha} \otimes \mathbf{v}\right)=\pi_{\alpha}^{i} X^{\alpha} \mathbf{v}_{i}
$$

Hence any horizontal Poisson $(n-1)$-form on $\Pi$ is the sum of: a form $\theta_{X}$ with $X$ a vertical vector field; the pull-back to $\Pi$ of an arbitrary horizontal $(n-1)$-form $\omega$ on $Y$; and a closed horizontal $(n-1)$-form $\mathrm{Z}$ on $\Pi$, that is

$$
F=\theta_{X}+\pi_{Y \Pi}^{*} \omega+Z \text {. }
$$

\section{Remarks.}

1. In classical mechanics (where $n=1, Y=\mathbb{R} \times Q$ and $M=\mathbb{R}$, with $Q$ an arbitrary manifold), although any function is a Poisson function, the previous Proposition gives a special kind of functions. The three terms are of the form

$$
F=\theta(X)+G(q, t)+k
$$

where $X$ is any vector in $\mathbb{R} \times Q, \theta$ is the Liouville form, $G$ is any function on $\mathbb{R} \times Q$ and $k$ is a constant which can be dropped. If $\left(q^{i}\right)$ is a coordinate system on $Q$, the local expression of these functions reads

$$
F=f^{i}(q, t) p_{i}+g(q, t)
$$

for time depending arbitrary functions $f^{i}$ and $g$ on $Q$, that is, we have the affine functions on $T^{*} Q$. It is interesting to point out that the set of these functions represents a natural class on which the Poisson bracket can be defined functorally and, are enough to determine the full Poisson bracket on the cotangent bundle as it is discussed in, for instance [6].

2. The notion of Poisson form as we present here follows the work of Kanatchikov [18], where the basic definitions are presented in coordinates.

3. The definition of Poisson forms can be extended to non-horizontal forms as it is done in, for example, see [9]. Nevertheless, the equations become much more complicated and hence, for our purposes, working with horizontal forms will be enough.

\section{The Poisson bracket and Hamilton equations}

Let $F$ be a horizontal Poisson $(n-1)$-form on $\Pi$ and $\mathcal{H}$ a Hamiltonian density. For the fixed volume form $\mathbf{v}$, we write, as before, $\mathcal{H}=H \mathbf{v}$. It is easy to check the following 
expression for the bracket:

$$
\{F, H\}=\frac{\partial F^{i}}{\partial y^{\alpha}} \frac{\partial H}{\partial \pi_{\alpha}^{i}}-\frac{\partial F^{i}}{\partial \pi_{\alpha}^{i}} \frac{\partial H}{\partial y^{\alpha}}
$$

in a coordinate system where $\mathbf{v}=\mathrm{d} x^{1} \wedge \cdots \wedge \mathrm{d} x^{n}$.

\section{Remarks.}

1. It is clear that the previous bracket depends on the choice of the volume form and it is not an intrinsic object of the Hamiltonian density $\mathcal{H}$. If a new volume form $\tilde{\mathbf{v}}=f \mathbf{v}, f \in$ $C^{\infty}(M)$, is chosen, we have $H=f \tilde{H}$ and hence $\{F, \tilde{H}\}=f\{F, H\}$. This dependence on $\mathbf{v}$ can be fixed by considering

$$
\{F, H\} \mathbf{v},
$$

instead of simply $\{F, H\}$. We can thus think of $\{F, H\} \mathbf{v}$ as the bracket of the Poisson form $F$ with the Hamiltonian density $\mathcal{H}$. Its local expression reads

$$
\{F, H\} \mathbf{v}:=\left(\frac{\partial F^{i}}{\partial y^{\alpha}} \frac{\partial H}{\partial \pi_{\alpha}^{i}}-\frac{\partial F^{i}}{\partial \pi_{\alpha}^{i}} \frac{\partial H}{\partial y^{\alpha}}\right) \mathrm{d} x^{1} \wedge \cdots \wedge \mathrm{d} x^{n} .
$$

2. The right-hand side of formula (5.1) does not depend on the chosen coordinate system and can be defined for any function $H$ and any horizontal form $F$, no matter whether $F$ is Poisson or not. That is, the bracket can be defined "geometrically" only for some special forms, but its local expression is intrinsic for any arbitrary form. This fact is related with the results in [6] where the Poisson bracket of mechanics is defined naturally only for a special class of functions and then extended to arbitrary functions.

Proposition 5.1. Given a connection $\mathcal{A}$ on $Y \rightarrow M$ and a Riemannian connection on $M$, there is a canonical connection on the bundle $\Pi \rightarrow M$. The horizontal lift of this connection is

$$
\frac{\partial}{\partial x^{i}} \mapsto \frac{\partial}{\partial x^{i}}+\Gamma_{i}^{\alpha} \frac{\partial}{\partial y^{\alpha}}+\left(-\frac{\partial \Gamma_{i}^{\beta}}{\partial y^{\alpha}} \pi_{\beta}^{j}+\Gamma_{i j}^{k} \pi_{\alpha}^{j}-\Gamma_{i k^{k}}^{k} \pi_{\alpha}^{j}\right) \frac{\partial}{\partial \pi_{\alpha}^{j}},
$$

where $\Gamma_{i}^{\alpha}$ are the coefficients of $\mathcal{A}$ (see formula (3.4)) and $\Gamma_{j k}^{i}$ are the Christoffel symbols of the Riemannian connection.

Proof. We first give a connection to the bundle $V^{*} Y \rightarrow M$. We understand the connection $\mathcal{A}$ as a section $\hat{\mathcal{A}}: Y \rightarrow J^{1} Y$ of the jet bundle. The differential of this map when restricted to vertical vectors is $\left.\mathrm{d} \hat{\mathcal{A}}\right|_{V Y}: V Y \rightarrow V J^{1} Y$. In the theory of jet bundles it is known that $V J^{1} Y$ and $J^{1} V Y$ are canonically isomorphic (for example, see [28]). Hence we have $\left.\mathrm{d} \hat{\mathcal{A}}\right|_{V Y}$ : $V Y \rightarrow J^{1} V Y$, that is a connection on the bundle $V Y \rightarrow M$. By duality we have a connection on $V^{*} Y \rightarrow M$. It is not difficult to prove that the horizontal lift of these connections are, respectively,

$$
\frac{\partial}{\partial x^{i}} \mapsto \frac{\partial}{\partial x^{i}}+\Gamma_{i}^{\alpha} \frac{\partial}{\partial y^{\alpha}}+\frac{\partial \Gamma_{i}^{\beta}}{\partial y^{\alpha}} \dot{y}^{\alpha} \frac{\partial}{\partial \dot{y}^{\beta}}, \quad \frac{\partial}{\partial x^{i}} \mapsto \frac{\partial}{\partial x^{i}}+\Gamma_{i}^{\alpha} \frac{\partial}{\partial y^{\alpha}}-\frac{\partial \Gamma_{i}^{\beta}}{\partial y^{\alpha}} p_{\beta} \frac{\partial}{\partial p_{\alpha}} .
$$


As $\Pi=T M \otimes_{Y} V^{*} Y \otimes_{Y} \bigwedge^{n} T^{*} M$, combining the connection on $V^{*} Y$ with the connections on $T M$ and $\bigwedge^{n} T^{*} M$ defined by the Riemannian connection gives a connection on $\Pi$ which is easily proved to have the local expression given in (5.2).

Theorem 5.2. A section $\pi$ of the bundle $\Pi \rightarrow M$ is a solution of a given Hamiltonian system $(\Pi, \mathcal{A}, \mathcal{H}), \mathcal{H}=H \mathbf{v}$, if and only if for any horizontal Poisson $(n-1)$-form $F$ the following equation holds true:

$$
\{F, H\} \mathbf{v} \circ \pi=\mathrm{d}\left(\pi^{*} F\right)-\left(\mathrm{d}^{\mathrm{h}} F\right) \circ \pi,
$$

where $\mathrm{d}^{\mathrm{h}} F$ is the horizontal differential of $F$, that is, the differential of $F$ restricted to horizontal vectors, with respect to the connection on $\Pi$, as was introduced in Proposition 5.1 .

Proof. In a coordinate system such that $\mathbf{v}=\mathrm{d} x^{1} \wedge \cdots \wedge \mathrm{d} x^{n}$, we have

$$
\{F, H\} \mathbf{v}=\left(\frac{\partial F^{i}}{\partial y^{\alpha}} \frac{\partial H}{\partial \pi_{\alpha}^{i}}-\frac{\partial F^{i}}{\partial \pi_{\alpha}^{i}} \frac{\partial H}{\partial y^{\alpha}}\right) \mathrm{d} x^{1} \wedge \cdots \wedge \mathrm{d} x^{n},
$$

and

$$
F=\left(-\pi_{\alpha}^{i} X^{\alpha}+g^{i}\right) \mathbf{v}_{i}
$$

Then

$$
\{F, H\} \mathbf{v}=\left(\frac{\partial F^{i}}{\partial y^{\alpha}} \frac{\partial H}{\partial \pi_{\alpha}^{i}}-X^{\alpha} \frac{\partial H}{\partial y^{\alpha}}\right) \mathrm{d} x^{1} \wedge \cdots \wedge \mathrm{d} x^{n} .
$$

On the other hand,

$$
\begin{aligned}
\mathrm{d}\left(\pi^{*} F\right)-\left(\mathrm{d}^{\mathrm{h}} F\right) \circ \pi= & \left(\frac{\partial F^{i}}{\partial x^{i}}+\frac{\partial F^{i}}{\partial y^{\alpha}} \frac{\partial y^{\alpha}}{\partial x^{i}}+\frac{\partial F^{i}}{\partial \pi_{\alpha}^{j}} \frac{\partial \pi_{\alpha}^{j}}{\partial x^{i}}-\frac{\partial F^{i}}{\partial x^{i}}-\Gamma_{i}^{\alpha} \frac{\partial F^{i}}{\partial y^{\alpha}}\right. \\
& \left.-\left(-\frac{\partial \Gamma_{i}^{\beta}}{\partial y^{\alpha}} \pi_{\beta}^{j}+\Gamma_{i k}^{j} \pi_{\alpha}^{k}-\Gamma_{i k}^{k} \pi_{\alpha}^{j}\right) \frac{\partial F^{i}}{\partial \pi_{\alpha}^{j}}\right) \mathbf{v} .
\end{aligned}
$$

But taking into account that $F$ is Poisson, from (4.4) we have

$$
\frac{\partial F^{i}}{\partial \pi_{\alpha}^{j}}=\delta_{j}^{i} X^{\alpha}
$$

and then

$$
\mathrm{d}\left(\pi^{*} F\right)-\left(\mathrm{d}^{\mathrm{h}} F\right) \circ h=\left(\frac{\partial F^{i}}{\partial y^{\alpha}}\left(\frac{\partial y^{\alpha}}{\partial x^{i}}-\Gamma_{i}^{\alpha}\right)+X^{\alpha}\left(\frac{\partial \pi_{\alpha}^{i}}{\partial x^{i}}+\frac{\partial \Gamma_{i}^{\beta}}{\partial y^{\alpha}} \pi_{\beta}^{i}\right)\right) \mathbf{v} .
$$

Hence (5.3) is true if and only if

$$
\left(\frac{\partial H}{\partial \pi_{\alpha}^{i}}\right)_{\pi}=\left(\frac{\partial y^{\alpha}}{\partial x^{i}}-\Gamma_{i}^{\alpha}\right)_{\pi}, \quad\left(\frac{\partial H}{\partial y^{\alpha}}\right)_{\pi}=-\left(\frac{\partial \pi_{\alpha}^{i}}{\partial x^{i}}+\frac{\partial \Gamma_{i}^{\beta}}{\partial y^{\alpha}} \pi_{\beta}^{i}\right)_{\pi},
$$

which are the Hamilton-de Donder equations, thus finishing the proof. 


\section{Remarks.}

1. We must note that the Riemannian connection defined on $M$ does not play an important role as the Christoffel symbols $\Gamma_{j k}^{i}$ do not appear in the computation of $\mathrm{d}(F \circ \pi)-\left(\mathrm{d}^{\mathrm{h}} F\right) \circ \pi$ for Poisson forms. In other words, for these forms the horizontal differential can be defined directly by means of the connection $\mathcal{A}$ only, without the use of any Riemannian connection.

2. In mechanics, where $M=\mathbb{R}$ and $n=1$, for the trivial connection on $\mathbb{R} \times Q \rightarrow \mathbb{R}$, the expression (5.3) simply reads

$$
\{F, H\} \mathrm{d} t=\left(\frac{\mathrm{d} F}{\mathrm{~d} t}-\frac{\partial F}{\partial t}\right) \mathrm{d} t,
$$

which is the classical formula of the Hamilton equations in the Poisson form.

\section{Lie-Poisson reduction in principal bundles}

We now confine ourselves to the case when the phase bundle is a $G$-principal bundle $\pi_{M P}: P \rightarrow M$. The goal of this section is the reduction of the Poisson equations (5.3) when the Hamiltonian density is invariant under the action of the full group $G$. The result of this reduction is the covariant version for field theories of the well-known Lie-Poisson equations in mechanics which represents the Poisson picture of the reduction principal bundles described in [2].

The space $T M \otimes \tilde{\mathfrak{g}}^{*} \otimes \bigwedge^{n} T^{*} M$. The right $G$-action on $P$ induces a natural action on $V P$ as a subset of $T P$. It is known that the quotient is isomorphic to the adjoint bundle, that is

$$
\frac{V P}{G} \simeq \tilde{\mathfrak{g}}
$$

Similarly, $\left(V^{*} P / G\right) \simeq \tilde{\mathfrak{g}}^{*}$. As the action of $G$ on $\Pi=T M \otimes_{P} V^{*} P \otimes_{P} \bigwedge^{n} T^{*} M$ is trivial on the factors $T M$ and $\bigwedge^{n} T^{*} M$, we thus have

$$
\frac{\Pi}{G} \simeq T M \otimes \tilde{\mathfrak{g}}^{*} \otimes \bigwedge^{n} T^{*} M .
$$

We now work with horizontal Poisson $(n-1)$-forms on $\Pi$ which are $G$-invariant. From Proposition 4.3 we know that such a form is of the type $F=\theta_{X}+\pi_{P \Pi}^{*} \omega+Z$, with $X$ a vertical vector field on $P, \omega \in \Omega^{n-1}(P)$ horizontal and $Z \in \Omega^{n-1}(\Pi)$ horizontal and closed. If we want $F$ to be $G$-invariant we thus have to deal with $G$-invariant vertical vector fields. Those vector fields are precisely the gauge vector fields of the principal bundle and they form a subalgebra of $\mathfrak{X}(P)$ denoted by gau $P$. It is well known that a gauge vector field can be seen as sections of the adjoint bundle $\tilde{\mathfrak{g}} \rightarrow M$, that is gau $P \simeq \Gamma(\tilde{\mathfrak{g}})$.

Proposition 6.1. The Poisson $(n-1)$-forms on $\Pi$ which are invariant under the action of $G$ on $\Pi$ are of the type

$$
F=\theta_{X}+\pi_{X \Pi}^{*} \omega+Z
$$

where $X \in \operatorname{gau} P, \omega \in \Omega^{n-1}(M)$ and $Z$ is a closed horizontal $G$-invariant form on $\Pi$. 
Proof. This is a straightforward computation.

The projection of the $G$-invariant Poisson $(n-1)$-forms on

$$
\frac{\Pi}{G}=T M \otimes \tilde{\mathfrak{g}}^{*} \otimes \bigwedge^{n} T^{*} M
$$

can be understood as follows. Given a gauge vector field on $P$, let $\xi$ be the section of $\tilde{\mathfrak{g}}$ determined by the identification gau $P \simeq \Gamma(\tilde{\mathfrak{g}})$. We define a mapping

$$
T M \otimes \tilde{\mathfrak{g}}^{*} \otimes \bigwedge^{n} T^{*} M \rightarrow T M \otimes \bigwedge^{n} T^{*} M
$$

by pairing the factor $\tilde{\mathfrak{g}}^{*}$ with $\xi$. By composing by the natural contraction $T M \otimes \bigwedge^{n} T^{*} M \rightarrow$ $\bigwedge^{n-1} T^{*} M$ we obtain a mapping

$$
\theta_{\xi}: T M \otimes \tilde{\mathfrak{g}}^{*} \otimes \bigwedge^{n} T^{*} M \rightarrow \bigwedge^{n-1} T^{*} M
$$

that is, an $(n-1)$-horizontal form on $\Pi / G$. In local coordinates, for $\xi=\xi^{\alpha} \tilde{B}_{\alpha}$, we have

$$
\mu_{\alpha}^{i} \frac{\partial}{\partial x^{i}} \otimes \tilde{B}^{\alpha} \otimes \mathbf{v} \mapsto \mu_{\alpha}^{i} \xi^{\alpha} \mathbf{v}_{i}
$$

Then it is easy to see the following proposition.

Proposition 6.2. Following the characterization given in Proposition 6.1, the forms $f$ on $\Pi / G$ which are projection of $G$-invariant horizontal Poisson $(n-1)$-forms on $\Pi$ are of the type

$$
f=\theta_{\xi}+\pi_{M \Pi / G}^{*} \omega+Z,
$$

where $\xi$ is any section of $\tilde{\mathfrak{g}} \rightarrow M$; and $\omega$ is any $(n-1)$-form on $M$ and $Z$ is any closed horizontal form on $\Pi / G$. Dropping the last term, the local expression of $f$ is

$$
f=f^{i} \mathbf{v}_{i}=\left(\xi^{\alpha} \mu_{\alpha}^{i}+\omega^{i}\right) \mathbf{v}_{i}
$$

The Lie-Poisson bracket. We can define a natural bracket on $\Pi / G=T M \otimes \tilde{\mathfrak{g}}^{*} \otimes$ $\bigwedge^{n} T^{*} M$ between functions $h$ and forms $f$ of the type described in Proposition 6.2 by means of the Lie coalgebra structure of the bundle $\tilde{\mathfrak{g}}^{*} \rightarrow M$. Given any function $h \in$ $C^{\infty}\left(T M \otimes \tilde{\mathfrak{g}}^{*} \otimes \bigwedge^{n} T^{*} M\right)$ its vertical derivative is a morphism

$$
\frac{\delta h}{\delta \mu}: T M \otimes \tilde{\mathfrak{g}}^{*} \otimes \bigwedge^{n} T^{*} M \rightarrow T^{*} M \otimes \tilde{\mathfrak{g}} \otimes \bigwedge^{n} T M
$$

defined by

$$
\frac{\delta h}{\delta \mu}(\mu)(\tau)=\left.\frac{\mathrm{d}}{\mathrm{d} \varepsilon}\right|_{\varepsilon=0} h(\mu+\varepsilon \tau)
$$


for any $\mu, \tau \in T_{x} M \otimes \tilde{\mathfrak{g}}_{x}^{*} \otimes \bigwedge^{n} T_{x}^{*} M, x \in M$. For any form $f$ of the type

$$
f=\theta_{\xi}+\pi_{M \Pi / G}^{*} \omega
$$

with $\xi \in \Gamma(\tilde{\mathfrak{g}})$, we define the Lie-Poisson brackets on $\Pi / G$ as

$$
\{f, h\}_{ \pm}(\mu)= \pm\left\langle\mu,\left[\xi, \frac{\delta h}{\delta \mu}(\mu)\right]\right\rangle
$$

for any $\mu \in T_{x} M \otimes \tilde{\mathfrak{g}}_{x}^{*} \otimes \bigwedge^{n} T_{x}^{*} M$, where the bracket [, ] is the fiberwise bracket in $\tilde{\mathfrak{g}} \rightarrow M$ and $\langle$,$\rangle stands for the natural pairing between T M \otimes \tilde{\mathfrak{g}}^{*} \otimes \bigwedge^{n} T^{*} M$ and $T^{*} M \otimes \tilde{\mathfrak{g}} \otimes \bigwedge^{n} T M$. Note that the Lie-Poisson bracket gives a function. The local expression of this bracket is

$$
\{f, h\}_{ \pm}(\mu)= \pm c_{\beta \gamma}^{\alpha} \mu_{\alpha}^{i} \xi^{\beta} \frac{\partial h}{\partial \mu_{\gamma}^{i}}= \pm c_{\beta \gamma}^{\alpha} \mu_{\alpha}^{i} \frac{\partial f^{i}}{\partial \mu_{\beta}^{i}} \frac{\partial h}{\partial \mu_{\gamma}^{i}},
$$

where $c_{\beta \gamma}^{\alpha}$ are the structure constants of the Lie algebra $\mathfrak{g}$.

\section{Remarks.}

1. As it is discussed in the first Remark in Section 5, if one is dealing with densities $\mathrm{h}=h \mathbf{v}$ on $T M \otimes \tilde{\mathfrak{g}}^{*} \otimes \bigwedge^{n} T^{*} M$ instead of with functions $h$, the Lie-Poisson bracket depends on the choice of the volume form $\mathbf{v}$. For that reason, we can define the Lie-Poisson bracket of forms and densities by simply writing

$$
\{f, h\}_{ \pm} \mathbf{v}
$$

which is a horizontal $n$-form on $\Pi / G$.

2. It is easy to check that the local expression (6.3) is intrinsic and hence one can define the bracket $\{f, g\}_{ \pm}$for any $(n-1)$-forms $f$ and $g$ non-necessarily of the form given in Proposition 6.2. This is related with the analogous fact commented in Remark 2 of Section 5.

3. For the case of mechanics, that is $P=\mathbb{R} \times Q \rightarrow \mathbb{R}$, the bracket defined by the formula (6.2) is nothing but the classical Lie-Poisson bracket of functions, as a simple computation shows, see, for example [21].

Covariant Lie-Poisson reduction. We now study the relationship between the Lie-Poisson bracket defined on $\Pi / G$ and the bracket given in $\Pi$ when dealing with $G$-invariant functions and $(n-1)$-Poisson forms.

Theorem 6.3. Let $\pi_{M P}: P \rightarrow M$ be a principal bundle with structure group $G$. Then, given an $(n-1)$-form $f$ as in $(6.1)$ and a function $h$ on $\Pi / G$, if $\kappa: \Pi \rightarrow \Pi / G$ represents the projection, we have

$$
\left\{\kappa^{*} f, \kappa^{*} h\right\}=\kappa^{*}\{f, h\}_{+},
$$

where $\{$,$\} is the bracket defined on \Pi$ by the multisymplectic form (see Section 4$)$ and $\{,\}_{+}$ is the Lie-Poisson bracket on $\Pi / G=T M \otimes \tilde{\mathfrak{g}}^{*} \otimes \bigwedge^{n} T^{*} M$ defined by formula (6.2). 
Proof. The formula we want to prove being local, we consider that the principal bundle is trivial, that is $P \simeq M \times G$. For any point $p \in P$ we can choose a trivialization such that $p=(x, e)$. Let be $\left\{B_{1}, \ldots, B_{m}\right\}$ be a basis of $\mathfrak{g}$. Let $\left(x^{i}, y^{\alpha}, \pi_{\alpha}^{i}\right)$ be a normal coordinate system in a neighborhood of $(x, e) \in P$ such that $\mathbf{v}=\mathrm{d}^{1} \wedge \cdots \wedge \mathrm{d} x^{n}$. Normal means that we define it by means of the exponential, more precisely, for $(x, g)$, the coordinates $y^{\alpha}(g)$ are such that

$$
g=\exp \left(y^{\alpha}(g) B_{\alpha}\right) .
$$

We denote as usual by $\left(x^{i}, y^{\alpha}, \pi_{\alpha}^{i}\right)$ the induced coordinate system on $\Pi$. For the proof of the theorem we need the following lemma.

Lemma 6.4. If $E$ is a $G$-invariant function on $\Pi$, with the above coordinate system we have

$$
\frac{\partial E}{\partial y^{\alpha}}=\frac{1}{2} c_{\beta \alpha}^{\gamma} \pi_{\gamma}^{j} \frac{\partial E}{\partial \pi_{\beta}^{j}},
$$

where $c_{\alpha \beta}^{\gamma}$ are the structure constants of the basis $\left\{B_{1}, \ldots, B_{m}\right\}$.

We continue the proof of the theorem. Let us call $H=h \circ \kappa$ and $F=\kappa^{*} f, F=$ $F^{i}\left(x^{j}, y^{\alpha}, \pi_{\alpha}^{j}\right) \mathbf{v}_{i}$. Note that either $F^{i}$ and $H$ are $G$-invariant. Hence, the local expression of the bracket reads

$$
\{F, H\} \mathbf{v}=\left(\frac{\partial F^{i}}{\partial y^{\alpha}} \frac{\partial H}{\partial \pi_{\alpha}^{i}}-\frac{\partial F^{i}}{\partial \pi_{\alpha}^{i}} \frac{\partial H}{\partial y_{\alpha}}\right) \mathbf{v}=\frac{1}{2}\left(\frac{\partial F^{i}}{\partial \pi_{\beta}^{j}} c_{\beta \alpha}^{\gamma} \pi_{\gamma}^{i} \frac{\partial H}{\partial \pi_{\alpha}^{i}}-\frac{\partial F^{i}}{\partial \pi_{\alpha}^{i}} \frac{\partial H}{\partial \pi_{\beta}^{j}} c_{\beta \alpha^{\gamma}}^{\pi_{\gamma}^{j}}\right) \mathbf{v},
$$

and taking into account formula (4.4)

$$
\{F, H\} \mathbf{v}=\frac{1}{2}\left(X^{\beta} c_{\beta \alpha}^{\gamma} \pi_{\gamma}^{i} \frac{\partial H}{\partial \pi_{\alpha}^{i}}-X^{\alpha} \frac{\partial H}{\partial \pi_{\beta}^{j}} c_{\beta \alpha}^{\gamma} \pi_{\gamma}^{j}\right) \mathbf{v}=X^{\beta} c_{\beta \alpha}^{\gamma} \pi_{\gamma}^{i} \frac{\partial H}{\partial \pi_{\alpha}^{i}} \mathbf{v},
$$

which is exactly $\kappa^{*}\{f, h\}_{+}$.

Proof of Lemma 6.4. This proof is rather technical. It is basically the Baker-Campbell-Hausdorff formula, which says that, for $X, Y \in \mathfrak{g}$, one has

$$
\exp X \cdot \exp Y=\exp \left(\sum_{n=1}^{\infty} c_{n}(X: Y)\right)
$$

where $c_{n}$ are some coefficients, $c_{n}(X: Y) \in \mathfrak{g}^{(n)}=\overbrace{[\mathfrak{g},[\cdots,[\mathfrak{g}, \mathfrak{g}] \cdots]}^{n}$. In fact, we will only need the first two coefficients $c_{1}(X: Y)=X+Y$ and $c_{2}(X: Y)=(1 / 2)[X, Y]$. We will denote $c_{n}^{\tau}(X: Y)$ the coordinates of $c_{n}(X: Y)$ with respect to the basis $\left\{B_{1}, \ldots, B_{m}\right\}$ of $\mathfrak{g}$.

For any $g \in G$, we compute the expression of

$$
T_{g} R_{g^{-1}}\left(\frac{\partial}{\partial y^{\gamma}}\right)_{g}=W_{\gamma}^{\beta}(g)\left(\frac{\partial}{\partial y^{\beta}}\right)_{e},
$$


that is, we determine the $W_{\alpha}^{\beta}(g)$. Let $\left(\bar{y}^{1}, \ldots, \bar{y}^{m}\right)$ be the coordinates of $g$. Then

$$
\begin{aligned}
T_{g} R_{g^{-1}}\left(\frac{\partial}{\partial y^{\gamma}}\right)_{g} & =\left.T_{g} R_{g^{-1}} \frac{\mathrm{d}}{\mathrm{d} \varepsilon}\right|_{\varepsilon=0} \exp \left(\bar{y}^{\tau} B_{\tau}+\varepsilon B_{\tau}\right) \\
& =\left.\frac{\mathrm{d}}{\mathrm{d} \varepsilon}\right|_{\varepsilon=0} \exp \left(\bar{y}^{\tau} B_{\tau}+\varepsilon B_{\tau}\right) \exp \left(-\bar{y}^{\tau} B_{\tau}\right) \\
& =\left.\sum_{n=1}^{\infty} \frac{\mathrm{d}}{\mathrm{d} \varepsilon}\right|_{\varepsilon=0} c_{n}^{\beta}\left(\bar{y}^{\tau} B_{\tau}+\varepsilon B_{\gamma}:-\bar{y}^{\tau} B_{\tau}\right)\left(\frac{\partial}{\partial y^{\beta}}\right)_{e},
\end{aligned}
$$

where $c_{1}\left(\bar{y}^{\beta} B_{\beta}+\varepsilon B_{\gamma}:-\bar{y}^{\beta} B_{\beta}\right)=\varepsilon B_{\gamma}$ and $c_{2}\left(\bar{y}^{\beta} B_{\beta}+\varepsilon B_{\gamma}:-\bar{y}^{\beta} B_{\beta}\right)=-(1 / 2)\left[\varepsilon B_{\gamma}, \bar{y}^{\beta} B_{\beta}\right]$. That is

$$
W_{\gamma}^{\beta}(g)=\left.\sum_{n=1}^{\infty} \frac{\mathrm{d}}{\mathrm{d} \varepsilon}\right|_{\varepsilon=0} c_{n}^{\beta}\left(\bar{y}^{\tau} B_{\tau}+\varepsilon B_{\gamma}:-\bar{y}^{\tau} B_{\tau}\right) .
$$

Then the dual

$$
\left(T_{g} R_{g^{-1}}\right)^{*}\left(\mathrm{~d} y^{\alpha}\right)_{e}=W_{\beta}^{\alpha}(g)\left(\mathrm{d} y^{\gamma}\right)_{g} .
$$

Taking into account the previous formula, the $G$-action on $\Pi$ has the following local expression: it sends the point $(x, e, \varpi), \varpi \in T_{x} M \otimes T_{e}^{*} G \otimes \bigwedge^{n} T_{x}^{*} M$ to

$$
\left(x^{i} ; 0, \ldots, 0 ; \pi_{1}^{i}, \ldots, \pi_{m}^{i}\right)_{e} \mapsto\left(x^{i} ; \bar{y}^{1}, \ldots, \bar{y}^{m} ; W_{1}^{\rho} \pi_{\rho}^{i}, \ldots, W_{\rho}^{1} \pi_{m}^{i}\right) .
$$

If $E \in C^{\infty}(\Pi)$ is $G$-invariant, we have

$$
E\left(x^{i} ; 0, \ldots, 0 ; \pi_{1}^{i}, \ldots, \pi_{m}^{i}\right)=E\left(x^{i} ; \bar{y}^{1}, \ldots, \bar{y}^{m} ; W_{1}^{\rho} \pi_{\rho}^{i}, \ldots, W_{\rho}^{1} \pi_{m}^{i}\right) .
$$

Making $g_{\epsilon}=\exp \left(\epsilon B_{\alpha}\right.$ ) (that is $\bar{y}^{1}=0, \ldots, \bar{y}^{\alpha}=\epsilon, \ldots, \bar{y}^{m}=0$ ) and computing the derivative with respect to $\epsilon$ we have

$$
0=\frac{\partial E}{\partial y^{\alpha}}+\left.\frac{\partial E}{\partial \pi_{\beta}^{i}} \frac{\mathrm{d}}{\mathrm{d} \epsilon}\right|_{\epsilon=0} W_{\beta}^{\gamma} \pi_{\gamma}^{i} .
$$

But

$$
\begin{aligned}
\left.\frac{\mathrm{d}}{\mathrm{d} \epsilon}\right|_{\epsilon=0} W_{\beta}^{\gamma}\left(g_{\epsilon}\right) & =\left.\left.\frac{\mathrm{d}}{\mathrm{d} \epsilon}\right|_{\epsilon=0} \sum_{n=1}^{\infty} \frac{\mathrm{d}}{\mathrm{d} \varepsilon}\right|_{\varepsilon=0} c_{n}^{\gamma}\left(\epsilon B_{\alpha}+\varepsilon B_{\beta}:-\epsilon B_{\alpha}\right) \\
& =-c_{2}^{\gamma}\left(B_{\beta}: B_{\alpha}\right)=-c_{\beta \alpha}^{\gamma},
\end{aligned}
$$

and then

$$
0=\frac{\partial E}{\partial y^{\alpha}}-\frac{\partial E}{\partial \pi_{\beta}^{i}} c_{\beta \alpha}^{\gamma} \pi_{\gamma}^{i} .
$$

We now recall the idea of divergence of valued vector fields. Given a principal bundle $P \rightarrow M$ endowed with a connection $\mathcal{A}$, and an associate vector bundle $V \rightarrow M$, we can 
define a divergence operator $\operatorname{div}^{\mathcal{A}}$ sending sections of $T M \otimes V \rightarrow M$ to sections of $V \rightarrow M$. $\operatorname{div}^{\mathcal{A}}$ is the only $\mathbb{R}$-operator such that

$$
\operatorname{div}\langle\chi, \eta\rangle=\left\langle\operatorname{div}^{\mathcal{A}} \chi, \eta\right\rangle+\left\langle\chi, \nabla^{\mathcal{A}} \eta\right\rangle
$$

for any $\chi \in \Gamma(T M \otimes V)$ and any $\eta \in \Gamma\left(V^{*}\right)$, where div stands for the usual divergence, $\langle$, is the natural pairing and

$$
\nabla^{\mathcal{A}}: \Gamma\left(V^{*}\right) \rightarrow \Gamma\left(T^{*} M \otimes V^{*}\right)
$$

is the covariant derivative defined by the connection $\mathcal{A}$ on the dual vector bundle $V^{*} \rightarrow$ $M$. Note that the principal connection endows any associate vector bundle with a linear connection and hence a covariant derivative. In particular, for $V=\tilde{\mathfrak{g}}^{*}$, it is easy to see that the local expression of the $\operatorname{div}^{\mathcal{A}}$ operator is

$$
\operatorname{div}^{\mathcal{A}}\left(\mu_{\alpha}^{i} \frac{\partial}{\partial x^{i}} \otimes \tilde{B}^{\alpha}\right)=\left(\frac{\partial \mu_{\alpha}^{i}}{\partial x^{i}}+c_{\gamma \alpha}^{\beta} \Gamma_{i}^{\gamma} \mu_{\beta}^{i}\right) \otimes \tilde{B}^{\alpha},
$$

where $\left\{\tilde{B}^{1}, \ldots, \tilde{B}^{m}\right\}$ is the basis of sections of $\tilde{\mathfrak{g}}^{*}$ induced by a chosen basis $\left\{B_{1}, \ldots, B_{m}\right\}$ in $\mathfrak{g}$.

Theorem 6.5. Let $\pi_{M P}: P \rightarrow M$ be a G-principal fiber bundle where $n=\operatorname{dim} M$ and $\mathbf{v}$ is a chosen volume form on $M$. Let $\mathcal{A}$ be a principal connection on $P \rightarrow M$ and $\mathcal{H} a$ Hamiltonian density on $\Pi$ invariant under the action of $G$ on $\Pi$. The dropped density to $\Pi / G$ is denoted by $\mathrm{h}=h \mathbf{v}$. For any section $\pi$ of the bundle $\Pi \rightarrow M$ let $\mu$ be the reduced section of

$$
\Pi / G=T M \otimes \tilde{\mathfrak{g}}^{*} \otimes \bigwedge^{n} T^{*} M \rightarrow M .
$$

Then the following assertions are equivalent:

1. for every horizontal Poisson $(n-1)$-form $F$ on $\Pi$, the following identity holds true:

$$
\pi^{*}\{F, H\} \mathbf{v}=\mathrm{d}\left(\pi^{*} F\right)-\mathrm{d}^{\mathrm{h}} F \circ \pi,
$$

2. the section $\pi$ is a solution of the Hamiltonian system $(\Pi, \mathcal{A}, \mathcal{H})$, that is, the Hamilton-de Donder equations are satisfied,

3. for every horizontal Poisson $(n-1)$-form $f$ on $\Pi / G$, the following identity holds true:

$$
\mu^{*}\{f, h\}_{+} \mathbf{v}=\mathrm{d}\left(\mu^{*} f\right)-\mathrm{d}^{\mathrm{h}} f \circ \mu,
$$

4. the section $\mu$ satisfies the Lie-Poisson equations

$$
\operatorname{div}^{\mathcal{A}} \mu=\operatorname{ad}_{\delta h / \delta \mu}^{*} \mu .
$$

Proof. The equivalence $1 \Leftrightarrow 2$ is provided by Theorem 5.2.

To establish the equivalence $1 \Leftrightarrow 3$, because the projection

$$
\kappa: \Pi \rightarrow \frac{\Pi}{G}
$$


is Poisson, the left-hand sides of equations in points $1 \Leftrightarrow 3$ are equivalent. We therefore only need to compare the right-hand sides. The formulas being local, we may assume that the bundle is trivializable. In fact, we choose a trivialization $P=M \times G$ such that the section $s: M \rightarrow P=M \times G$ is the identity section $s(x)=(x, e)$. We identify $V P$ with $M \times T G$ and $\tilde{\mathfrak{g}}$ with a subset of $M \times T_{e} G=M \times \mathfrak{g}$ of $V P$. Similarly, $\tilde{\mathfrak{g}}^{*}$ is seen as a subset $M \times \mathfrak{g}^{*}$ of

$$
V^{*} P=M \times T^{*} G
$$

In a coordinate system $\left(x^{i}, y^{\alpha}\right)$ of $P$ such that $\mathbf{v}=\mathrm{d} x^{1} \wedge \cdots \wedge \mathrm{d} x^{n}$ and $\left(y^{\alpha}\right)$ is a normal coordinate system on $G$, taking into account (6.4), we have

$$
\begin{aligned}
\mathrm{d}\left(\pi^{*} F\right)-\mathrm{d}^{\mathrm{h}} F \circ h & \left(\frac{\partial F^{i}}{\partial x^{i}}+\frac{\partial F^{i}}{\partial y^{\alpha}} \frac{\partial y^{\alpha}}{\partial x^{i}}+\frac{\partial F^{i}}{\partial \pi_{\alpha}^{j}} \frac{\partial \pi_{\alpha}^{j}}{\partial x^{i}}-\frac{\partial F^{i}}{\partial x^{i}}-\frac{\partial F^{i}}{\partial y^{\alpha}} \Gamma_{i}^{\alpha}\right. \\
& \left.-\frac{\partial F^{i}}{\partial \pi_{\alpha}^{j}}\left(-\frac{\partial \Gamma_{i}^{\beta}}{\partial y^{\alpha}} \pi_{\beta}^{i}+\Gamma_{i k^{j}}^{j} \pi_{\alpha}^{k}-\Gamma_{i k}^{k} \pi_{\alpha}^{j}\right)\right) \mathbf{v} \\
= & \left(\frac{\partial F^{i}}{\partial x^{i}}+\frac{\partial F^{i}}{\partial \pi_{\alpha}^{j}} \frac{\partial \pi_{\alpha}^{j}}{\partial x^{i}}-\frac{\partial F^{i}}{\partial x^{i}}-\frac{1}{2} c_{\beta \alpha}^{\gamma} \pi_{\gamma}^{j} \frac{\partial F^{i}}{\partial \pi_{\beta}^{j}} \Gamma_{i}^{\alpha}\right. \\
& \left.-\frac{\partial F^{i}}{\partial \pi_{\alpha}^{j}}\left(-\frac{\partial \Gamma_{i}^{\beta}}{\partial y^{\alpha}} \pi_{\beta}^{j}+\Gamma_{i k^{j}}^{j} \pi_{\alpha}^{k}-\Gamma_{i k}^{k} \pi_{\alpha}^{j}\right)\right) \mathbf{v} \\
\stackrel{(*)}{=} & \left(\frac{\partial F^{i}}{\partial x^{i}}+\frac{\partial F^{i}}{\partial \pi_{\alpha}^{j}} \frac{\partial \pi_{\alpha}^{j}}{\partial x^{i}}-\frac{\partial F^{i}}{\partial x^{i}}-\frac{1}{2} c_{\beta \alpha^{\gamma}}^{\gamma} \pi_{\gamma}^{i} \frac{\partial F^{i}}{\partial \pi_{\beta}^{j}} \Gamma_{j}^{\alpha}\right. \\
& \left.-\frac{\partial F^{i}}{\partial \pi_{\alpha}^{j}}\left(-\frac{1}{2} c_{\gamma \alpha}^{\beta} \Gamma_{i}^{\gamma} \pi_{\beta}^{j}+\Gamma_{i k}^{j} \pi_{\alpha}^{k}-\Gamma_{i k}^{k} \pi_{\alpha}^{j}\right)\right) \\
= & \left(\frac{\partial F^{i}}{\partial x^{i}}+\frac{\partial F^{i}}{\partial \pi_{\alpha}^{j}} \frac{\partial \pi_{\alpha}^{j}}{\partial x^{i}}-\frac{\partial F^{i}}{\partial x^{i}}-\frac{\partial F^{i}}{\partial \pi_{\alpha}^{j}}\left(-c_{\gamma \alpha}^{\beta} \Gamma_{i}^{\gamma} \pi_{\beta}^{j}+\Gamma_{i k}^{j} \pi_{\alpha}^{k}-\Gamma_{i k}^{k} \pi_{\alpha}^{j}\right)\right) \mathbf{v} .
\end{aligned}
$$

For the step (*), as the connection $\mathcal{A}$ is a principal connection (that is, it is $G$-invariant), it is not difficult to adapt the proof of Lemma 6.4 to obtain

$$
\frac{\partial \Gamma_{i}^{\beta}}{\partial y^{\alpha}}=\frac{1}{2} c_{\gamma \alpha}^{\beta} \Gamma_{i}^{\gamma} .
$$

Taking into account that we are working along the identity section $s(x)=(x, e)$, the expression above evidently coincides with

$$
\begin{aligned}
& \mathrm{d} \mu^{*} f-\mathrm{d}^{\mathrm{h}} f \circ \mu \\
& \quad=\left(\frac{\partial f^{i}}{\partial x^{i}}+\frac{\partial f^{i}}{\partial \mu_{\alpha}^{j}} \frac{\partial \mu_{\alpha}^{j}}{\partial x^{i}}-\frac{\partial f^{i}}{\partial x^{i}}-\frac{\partial f^{i}}{\partial \mu_{\alpha}^{j}}\left(-c_{\gamma \alpha}^{\beta} \Gamma_{i}^{\gamma} \mu_{\beta}^{j}+\Gamma_{i k}^{j} \mu_{\alpha}^{k}-\Gamma_{i k}^{k} \mu_{\alpha}^{j}\right)\right) \mathbf{v} .
\end{aligned}
$$

Actually, we have not used the fact that $F$ is Poisson. The identity is valid for any projectable horizontal $(n-1)$-form on $\Pi$. 
For $3 \Leftrightarrow 4$, taking into account the structure of the form $f$ given in (6.1), the local expression on the left-hand side of (6.5) reads

$$
\begin{aligned}
& \mathrm{d} \mu^{*} f-\mathrm{d}^{\mathrm{h}} f \circ \mu \\
& =\left(\frac{\partial f^{i}}{\partial x^{i}}+\frac{\partial f^{i}}{\partial \mu_{\alpha}^{j}} \frac{\partial \mu_{\alpha}^{j}}{\partial x^{i}}-\frac{\partial f^{i}}{\partial x^{i}}-\frac{\partial f^{i}}{\partial \mu_{\alpha}^{j}}\left(-c_{\gamma \alpha}^{\beta} \Gamma_{i}^{\gamma} \mu_{\beta}^{j}+\Gamma_{i k}^{j} \mu_{\alpha}^{k}-\Gamma_{i k}^{k} \mu_{\alpha}^{j}\right)\right) \mathbf{v} \\
& =X^{\alpha}\left(\frac{\partial \mu_{\alpha}^{i}}{\partial x^{i}}+c_{\gamma \alpha}^{\beta} \Gamma_{i}^{\gamma} \mu_{\beta}^{i}\right) \mathbf{v}=\left\langle X, \operatorname{div}^{\mathcal{A}} \mu\right\rangle \mathbf{v} .
\end{aligned}
$$

Hence, taking into account the definition of the Lie-Poisson bracket $\{,\}_{+}$, Eq. (6.5) can be written as

$$
\left\langle\xi, \operatorname{ad}_{\delta h / \delta \mu}^{*} \mu\right\rangle=\left\langle\xi, \operatorname{div}^{\mathcal{A}} \mu\right\rangle
$$

for any $\xi \in \Gamma(\tilde{\mathfrak{g}})$, which is only possible if and only if (6.6) is satisfied.

\section{Remarks.}

1. Eq. (6.6) become the classical Lie-Poisson equation when $Y=\mathbb{R} \times Q$ and $\mathcal{A}$ is the flat connection, that is, the case of mechanics, see, for instance [21] for this classical result.

Euler-Poincaré and Lie-Poisson for hyper-regular Lagrangians. The Lagrangian picture of covariant reduction for principal bundles was studied in $[2,4]$. We now present the link between that theory and Theorem 6.5 when the Lagrangian density $\mathcal{L}$ is hyper-regular. First, we state the basic result of the Lagrangian reduction called Euler-Poincaré reduction.

Theorem 6.6. Let $\pi_{M P}: P \rightarrow M$ be a principal $G$-fiber bundle over a manifold $M$ with a volume form $\mathbf{v}$ and let $L: J^{1} P \rightarrow \mathbb{R}$ be $G$-invariant Lagrangian. Let $l: C \rightarrow \mathbb{R}$ be the mapping defined by $L$ on the quotient. For an open set $U \subset M$ with $\bar{U}$ compact and a section $s: \bar{U} \rightarrow P$ of $\pi$, define $\sigma: U \rightarrow C$ by $\sigma(x)=q\left(j_{x}^{1} s\right)$, where $q: J^{1} P \rightarrow C=\left(J^{1} P\right) / G$ is the canonical projection. Then, for every principal connection $\mathcal{A}$ on $P$, the following are equivalent:

1. the variational principle $\delta \int_{U} L\left(j_{x}^{1} s\right) \mathbf{v}=0$ holds, for vertical variations $\delta_{s}$ along $s$ with compact support,

2. the local section $s: M \rightarrow P$ satisfies the Euler-Lagrange equations for $L$,

3. the variational principle $\delta \int_{U} l(\sigma(x)) \mathbf{v}=0$ holds, using variations of the form

$$
\delta \sigma=\nabla^{\mathcal{A}} \eta-\left[\sigma^{\mathcal{A}}, \eta\right]
$$

where $\eta: M \rightarrow \tilde{\mathfrak{g}}$ is any section of the adjoint bundle with compact support, and $\sigma^{\mathcal{A}}$ is the section of $T^{*} M \otimes \tilde{\mathfrak{g}}$ such that $\sigma=\mathcal{A}+\sigma^{\mathcal{A}}$,

4. the Euler-Poincaré equations hold:

$$
\operatorname{div}_{\mathcal{A}} \frac{\delta l}{\delta \sigma}=\operatorname{ad}_{\sigma^{\mathcal{A}}}^{*} \frac{\delta l}{\delta \sigma}
$$

where $\delta l / \delta \sigma \in \Gamma\left(T M \otimes \tilde{\mathfrak{g}}^{*}\right)$ is the vertical differential of $l$ along $\sigma$. 
For the third point, note that $C \rightarrow M$ is an affine bundle modeled over the vector bundle $T^{*} M \otimes \tilde{\mathfrak{g}}$.

The dropped Lagrangian $l: C \rightarrow \mathbb{R}$ defines a dropped Legendre transformation

$$
\mathfrak{f} l: C \rightarrow T M \otimes \tilde{\mathfrak{g}}^{*}
$$

by setting

$$
\mathrm{f} l(\sigma)\left(\sigma^{\prime}\right)=\left.\frac{\mathrm{d}}{\mathrm{d} \varepsilon}\right|_{\varepsilon=0} l\left(\sigma+\varepsilon \sigma^{\prime}\right),
$$

that is, the vertical differential of $l$. In fact, in order to take into account the volume form $\mathbf{v}$, we can define $\widehat{\mathrm{f} l}: C \rightarrow \Pi / G=T M \otimes \tilde{\mathfrak{g}}^{*} \otimes \bigwedge^{n} T^{*} M$ by $\widehat{\mathrm{f} l}=\mathrm{f} l \mathbf{v}$. It is evident that, for a $G$-invariant Lagrangian density, the following diagram commutes:

$$
\begin{array}{rll}
J^{1} P & \stackrel{\widehat{F L}}{\longrightarrow} & \Pi \\
q \downarrow & & \downarrow \kappa \\
C & \stackrel{\widehat{\mathrm{f} l}}{\longrightarrow} & \Pi / G
\end{array}
$$

In fact, given a section $\sigma \in \Gamma(G)$, we have that

$$
\mu=\widehat{\mathfrak{f} l} \circ \sigma=\frac{\delta l}{\delta \sigma} \mathbf{v} .
$$

On the other hand, form the local expression of the Hamiltonian density $\mathcal{H}$ defined the connection $\mathcal{A}$ and the Lagrangian $\mathcal{L}$, it is clear that the "inverse Legendre transformation"

$$
\begin{aligned}
& \widehat{\mathbb{F H}}: \Pi \rightarrow T^{*} M \otimes V P \otimes \bigwedge^{n} T^{*} M, \\
& \widehat{\mathbb{F H}}(\varpi)\left(\varpi^{\prime}\right)=\left.\frac{\mathrm{d}}{\mathrm{d} \varepsilon}\right|_{\varepsilon=0} \mathcal{H}\left(\varpi+\varepsilon \varpi^{\prime}\right)
\end{aligned}
$$

induced by the Hamiltonian density satisfies

$$
\begin{aligned}
J^{1} P & \stackrel{\widehat{F L}}{\longrightarrow} \Pi \\
F_{A} \downarrow & \swarrow^{\widehat{F H}} \\
J^{1} P_{A} &
\end{aligned}
$$

where $J^{1} P_{\mathcal{A}}=T^{*} M \otimes V P \otimes \bigwedge^{n} T^{*} M$ and the identification $F_{\mathcal{A}}$ is, modulo the volume factor $\bigwedge^{n} T^{*} M$, the linearization of the affine bundle $J^{1} P \rightarrow P$ when the section $\hat{\mathcal{A}}: P \rightarrow J^{1} P$ defined by the connection is taken to be the zero section. Roughly speaking, $\widehat{\mathbb{F H}}$ is the inverse of $\widehat{\mathbb{F}}$ when the identification $F_{\mathcal{A}}$ is assumed. When the diagram (6.10) is reduced by the action of the group $G$ we have

$$
\begin{array}{ccc}
C & \stackrel{\widehat{\mathrm{fl}}}{\rightarrow} & \frac{\Pi}{G} \\
\downarrow & \swarrow^{\widehat{\mathrm{fh}}} & \\
C_{\mathcal{A}} & &
\end{array}
$$


where $C_{\mathcal{A}}=T^{*} M \otimes \tilde{\mathfrak{g}} \otimes \bigwedge^{n} T^{*} M$. When $\mu$ is a section of $\Pi / G \rightarrow M$, the value of $\widehat{\mathrm{f} h}$ along $\mu$ is just $\delta h / \delta \mu$ and hence, when $\mu=\widehat{\mathrm{f} l} \circ \sigma$ we have

$$
\frac{\delta h}{\delta \mu}=\sigma_{\mathcal{A}}
$$

Theorem 6.7. Let $\pi_{M P}: P \rightarrow M$ be a principal $G$-fiber bundle over a manifold $M$ with a volume form $\mathbf{v}$ and let $\mathcal{L}: J^{1} P \rightarrow \bigwedge^{n} T^{*} M$ be a G-invariant hyper-regular Lagrangian density, $\mathcal{L}=$ Lv. Let $l: C \rightarrow \mathbb{R}$ be the mapping defined by $L$ on the quotient. We endow $P \rightarrow M$ with a connection $\mathcal{A}$ on $P$ and we define the Hamiltonian density $\mathcal{H}=\mathcal{H}_{\mathcal{L}}^{\mathcal{A}}$ on $\Pi$ and the dropped Hamiltonian $\mathrm{h}=h \mathbf{v}$ on $\Pi / G$. For an open set $U \subset M$ with $\bar{U}$ compact and a section $s: \bar{U} \rightarrow P$ of $\pi_{M P}$, define $\sigma(x)=q \circ j_{x}^{1} s ; \pi=\widehat{\mathbb{F L}} \circ j^{1} s$ and $\mu=\kappa \circ \pi$. Then, for every principal connection $\mathcal{A}$ on $P$, the following are equivalent:

1. $s$ is a critical section of the variational problem defined by $\mathcal{L}$,

2. $\sigma$ is a solution of the Euler-Poincaré equations (6.8),

3. $\pi$ is a solution of the Hamiltonian equations defined by the connection $\mathcal{A}$ and the Hamiltonian density $\mathcal{H}$,

4. $\mu$ is a solution of the Lie-Poisson equations (6.6).

Proof. The equivalence $1 \Leftrightarrow 3$ is Theorem 3.4 . The reductions $3 \Leftrightarrow 4$ and $1 \Leftrightarrow 2$ are consequence of Theorems 6.5 and 6.6, respectively.

In fact, the equivalence of the Euler-Poincaré equations and Lie-Poisson for hyper-regular Lagrangians can be obtained from formulas (6.9) and (6.11).

\section{Electromagnetism}

Infinite dimensional reduction. The reduction of Maxwell's equations from the point of view of symplectic, Poisson and Lagrangian reduction in the infinite dimensional context is well known (see, for instance, $[21,24]$ ). The Poisson view starts with the canonical symplectic structure on the cotangent bundle $T^{*} Q$ of the space $Q$ of vector potentials A on $\mathbb{R}^{3}$. This Maxwell configuration space $Q$ has, as its cotangent bundle, the space $(\mathbf{A}, \mathbf{E})$ of vector potentials together with their conjugate momenta, the space of electric fields $\mathbf{E}$ (up to a sign).

The gauge group $\mathfrak{G}$ consists of all real valued functions $\phi$ on $\mathbb{R}^{3}$ with appropriate fall off conditions at infinity. One then takes the quotient of $T^{*} Q$ with respect to the action of the gauge group $\mathfrak{B}$ whose action on configuration space is simply $\mathbf{A} \mapsto \mathbf{A}+\nabla \phi$. The quotient of the canonical Poisson structure gives the Pauli-Born-Infeld (PBI) Poisson structure on the space of electric and magnetic fields $\mathbf{E}, \mathbf{B}$. The reduction process in this sort of example, which is due to [24], is discussed in, for example, [21]. We recall for the readers convenience that the PBI bracket is given on functions $f$ and $g$ of $(\mathbf{E}, \mathbf{B})$ by

$$
\{f, g\}=\iiint\left[\frac{\delta f}{\delta \mathbf{E}} \cdot\left(\nabla \times \frac{\delta g}{\delta \mathbf{B}}\right)-\frac{\delta g}{\delta \mathbf{E}} \cdot\left(\nabla \times \frac{\delta f}{\delta \mathbf{B}}\right)\right] \mathrm{d}^{3} x .
$$


Here it is assumed that the fields are defined on all of $\mathbb{R}^{3}$ for simplicity and where the derivatives are taken in the sense of functional derivatives.

The dynamic Maxwell equations are then the Hamilton equations for this Poisson structure together with the Hamiltonian

$$
H=\frac{1}{2} \iiint\left[\|\mathbf{E}\|^{2}+\|\mathbf{B}\|^{2}\right] \mathrm{d}^{3} x .
$$

This process is also important for understanding the Poisson structure of fields coupled to the Maxwell equations such as charged fluids and the Maxwell-Vlasov equations, see [21] for the literature and also [5].

Symplectic reduction. In this context, symplectic reduction is quite simple. Namely, the momentum map for the action of the gauge group is simply the divergence of the electric field, so the process of setting the momentum map to be a constant reproduces the Maxwell equation $\operatorname{div} \mathbf{E}=\rho$ and taking the quotient by the gauge group then maps $\mathbf{A}$ to $\mathbf{B}$. The reduced symplectic form is the one associated with the Born-Infeld Poisson structure on this space of $\mathbf{E}$ and $\mathbf{B}$.

Lagrangian reduction. Lagrangian reduction can be carried out following the general principles in [22] in a straightforward way. Namely, one starts with the same Maxwell configuration space $Q$ but now one constructs the tangent bundle $T Q$ of pairs $(\mathbf{A}, \dot{\mathbf{A}})$. We ultimately identify the time derivative of $\mathbf{A}$ (or rather its negative) with the electric field $\mathbf{E}$. We define $\mathbf{B}=\nabla \times \mathbf{A}$ and let the Lagrangian be

$$
L(\mathbf{A}, \dot{\mathbf{A}})=\frac{1}{2} \iiint\left[\|\dot{\mathbf{A}}\|^{2}-\|\nabla \times \mathbf{A}\|^{2}\right] \mathrm{d}^{3} x .
$$

One checks that the dynamic Maxwell equations are the Euler-Lagrange equations for this Lagrangian. Of course, the Euler-Lagrange equations are equivalent to Hamilton's principle, namely

$$
\delta \iiint L(\mathbf{A}, \dot{\mathbf{A}}) \mathrm{d}^{3} x=0 .
$$

Lagrangian reduction focuses on the reduction of Hamilton's principle. In this case, this procedure is particularly simple; we form the quotient space $T Q / \mathfrak{B}$, which we identify with the space of pairs $(\mathbf{B}, \mathbf{E})$ and define the reduced Lagrangian by the same expression as $L$ except now regarded as a function of $(\mathbf{B}, \mathbf{E})$ :

$$
l(\mathbf{B}, \mathbf{E})=\frac{1}{2} \iiint\left[\|\mathbf{E}\|^{2}-\|\mathbf{B}\|^{2}\right] \mathrm{d}^{3} x .
$$

The reduced variational principle now states that the previous variational principle and hence the Maxwell equations are equivalent to

$$
\delta \int l(\mathbf{B}, \mathbf{E}) \mathrm{d} t=0
$$

for variations of a given curve of fields $(\mathbf{B}(t), \mathbf{E}(t))$ that have the form

$$
\delta \mathbf{B}=\nabla \times \xi, \quad \delta \mathbf{E}=-\dot{\xi}
$$


for some curve of vector fields $\xi(t)$. Variations of this form are induced by variations of $\mathbf{A}$ via $\delta \mathbf{A}=\xi$.

Covariant Lagrangian reduction for electromagnetism. We now consider the configuration bundle $\pi_{T^{*}}: T^{*} M \rightarrow M$ over a semi-Riemannian manifold $(M, g)$ and the Lagrangian density

$$
\mathcal{L}: J^{1}\left(T^{*} M\right) \rightarrow \bigwedge^{n} T^{*} M, \quad \mathcal{L}\left(j_{x}^{1} \omega\right)=\frac{1}{2}\langle\mathrm{~d} \omega, \mathrm{d} \omega\rangle_{g} \mathbf{v}_{g},
$$

where $\langle,\rangle_{g}$ is the Riemannian metric defined by $g$ on $\bigwedge^{2} T^{*} M$ and $\mathbf{v}_{g}$ is the semi-Riemannian volume. In coordinates

$$
\mathcal{L}\left(j_{x}^{1} \omega\right)=\frac{1}{4} F_{\mu \nu} F^{\mu \nu} \sqrt{g} \mathrm{~d} x^{1} \wedge \cdots \wedge \mathrm{d} x^{n} .
$$

The bundle $T^{*} M \rightarrow M$ can be considered as the bundle of connections of the trivial bundle $M \times U(1) \rightarrow M$. In fact, representation of the gauge transformations on the bundle of connections are symmetries of the electromagnetic Lagrangian. More precisely, for any gauge transformation $\Psi: M \times U(1) \rightarrow M \times U(1), \Psi(x, z)=\left(x, \mathrm{e}^{\mathrm{i} \phi(x)} z\right)$, with $\phi \in C^{\infty}(M)$, the transformation $T^{*} M \rightarrow T^{*} M, \omega_{x} \mapsto \omega_{x}+(\mathrm{d} \phi)_{x}$, with $\omega_{x} \in T_{x}^{*} M$, leaves the Lagrangian $\mathcal{L}$ invariant. The mappings $\phi$ can be seen as sections of the trivial bundle $M \times \mathbb{R} \rightarrow M$. Hence, considering the jet bundle $J^{1}(M, \mathbb{R})$ we have a fiberwise action

$$
J^{1}(M, \mathbb{R}) \times T^{*} M \rightarrow T^{*} M, \quad\left(j_{x}^{1} \phi, \omega_{x}\right) \mapsto \omega_{x}+(\mathrm{d} \phi)_{x},
$$

which induces an action

$$
J^{2}(M, \mathbb{R}) \times J^{1}\left(T^{*} M\right) \rightarrow J^{1}\left(T^{*} M\right),
$$

by simply 1 -jet prolongation.

Proposition 7.1. The quotient space $J^{1}\left(T^{*} M\right) / J^{2}(M, \mathbb{R})$ can be naturally identified with $\bigwedge^{2} T^{*} M$ and the projection $J^{1}\left(T^{*} M\right) \rightarrow \bigwedge^{2} T^{*} M$ with the differential $j_{x}^{1} \omega \mapsto(\mathrm{d} \omega)_{x}$.

Proof. By virtue of Poincaré lemma, two 1 -jets $j_{x}^{1} \omega$ and $j_{x}^{1} \omega^{\prime}$ of local sections are mapped to the same 2-covector by the differential if and only if $\omega^{\prime}=\omega+\mathrm{d} \phi$ for a local function $\phi$. The prove is complete by taking into account that the differential morphism is a surjective fibration.

Since the electromagnetic Lagrangian $\mathcal{L}$ is invariant under the gauge transformation, it drops to the quotient space as a map

$$
\ell: \frac{J^{1}(Y)}{J^{2}(M, \mathbb{R})} \rightarrow \Lambda^{n} T^{*} M .
$$

This is a particular case of the Utiyama theorem (see, for example [11]).

Following the guide from the infinite dimensional space of fields point of view, one should consider variations of sections of the bundle $Y$ that are needed to form the Euler-Lagrange 
equations. These variations (probably one should start with vertical variations) project to variations on the covariant reduced space $J^{1}(Y) / J^{2}(M, \mathbb{R})=\bigwedge^{2} T^{*} M$ that are the covariant analog of the induced variations of the magnetic field we saw in the infinite dimensional point of view. Namely, the variations should be of the form of the linearization of the curvature operator applied to a variation of $A$.

We start with a section $A$ of $T^{*} M$ and we consider an arbitrary vertical variation $A_{\varepsilon}$ of the type $A_{\varepsilon}=A+\varepsilon \omega$, where $\omega$ is another 1-form, that is, we have $\delta A=\omega$. The dropped variation will be

$$
\left.\frac{\mathrm{d}}{\mathrm{d} \varepsilon}\right|_{\varepsilon=0} \mathrm{~d} A_{\varepsilon}=\mathrm{d} \omega .
$$

Hence the infinitesimal variation along $F=\mathrm{d} A$ will be $\delta F=\mathrm{d} \omega$ and we can say that the reduced problem is a zero-order variational problem defined by the Lagrangian $l: \bigwedge^{2}(X, \tilde{\mathfrak{g}}) \rightarrow \mathbb{R}, l(F)=(1 / 2)\|F\|^{2}$, under constraints: the admissible variations along a section $F$ are of the type $\mathrm{d} \omega$, with $\omega \in \Omega^{1}(X)$. The variational principle yields

$$
\begin{aligned}
0 & =\delta \int_{X} l(F) \mathbf{v}_{g}=\left.\frac{1}{2} \frac{\mathrm{d}}{\mathrm{d} \varepsilon}\right|_{\varepsilon=0} \int_{X}\|F+\varepsilon \mathrm{d} \omega\|^{2} \mathbf{v}_{g} \\
& =\left.\frac{1}{2} \frac{\mathrm{d}}{\mathrm{d} \varepsilon}\right|_{\varepsilon=0} \int_{X}\langle F+\varepsilon \mathrm{d} \omega, F+\varepsilon \mathrm{d} \omega\rangle \mathbf{v}_{g}=\int_{X}\langle F, \mathrm{~d} \omega\rangle \mathbf{v}_{g}=\int_{X}\langle\partial F, \omega\rangle \mathbf{v}_{g},
\end{aligned}
$$

where $\partial F$ is the codifferential defined by the metric $g$. As $\omega$ is arbitrary, the variational principle gives

$$
\partial F=0
$$

which is one half of the Maxwell equations. The other half comes from the compatibility conditions for reconstruction. Locally, a 2-form is the differential of a 1-form $A$ if and only if $F$ is exact. Hence a necessary condition for reconstruction is

$$
\mathrm{d} F=0 .
$$

These results for electromagnetism are similar to those of Fernández et al. [8].

Almost regular Lagrangians. We pause momentarily to recall a bit more of the general theory. Given a fiber bundle $Y \rightarrow M$, a Lagrangian density $\mathcal{L}$ is said to be almost regular if the following properties are satisfied (see, for example [7,12]):

1. $\widehat{\mathbb{F} \mathcal{L}}\left(J^{1} Y\right) \subset \Pi$ is a closed submanifold.

2. $\widehat{\mathbb{F L}}$ is a submersion with connected fibers.

The manifold $\mathcal{P}=\widehat{\mathbb{F L}}\left(J^{1} Y\right)$ is called the constraint manifold. For $\kappa \circ \mathbb{F} \mathcal{L}=\widehat{\mathbb{F L}}$, we have that $\mathbb{F} \mathcal{L}\left(J^{1} Y\right) \subset \kappa^{-1}(\mathcal{P})$ and we can define a section $\delta$ of $\kappa$ along $\mathcal{P}$ by setting $\delta=\mathbb{F} \mathcal{L} \circ \widehat{\mathbb{F L}}^{-1}$. Note that $\widehat{\mathbb{F L}}^{-1}(\varpi), \varpi \in \mathcal{P}$, is a submanifold of $J^{1} Y$ on which $\mathbb{F} \mathcal{L}$ is constant and hence the definition $\delta=\mathbb{F} \mathcal{L} \circ \widehat{\mathbb{F L}}^{-1}$ makes sense. Using a 
diagram

$$
\begin{array}{cccccc}
J^{1} Y & \stackrel{\mathbb{F} \mathcal{L}}{\rightarrow} & \kappa^{-1}(\mathcal{P}) & \hookrightarrow & J^{1} Y^{*} \\
\| & & \kappa \downarrow \uparrow \delta & & \downarrow \kappa \\
J^{1} Y & \stackrel{\mathbb{F L}}{\nrightarrow} & \mathcal{P} & \hookrightarrow & \Pi \\
& & \downarrow & & \downarrow \\
& & M & = & M
\end{array}
$$

A pair $(\mathcal{P}, \delta)$ of a subbundle $\mathcal{P}$ of $\Pi \rightarrow Y$ and a section of $\kappa: J^{1} Y^{*} \rightarrow \Pi$ along $\mathcal{P}$ is called a Hamiltonian system with constraints. A section $\pi$ of $\mathcal{P} \rightarrow M$ is called a solution if

$$
\pi^{*}\left(\mathbf{i}_{X} \Omega_{\delta}^{\mathcal{P}}\right)=0
$$

for any vertical vector $X$, where $\Omega^{\mathcal{P}}=\mathrm{d} \Theta^{\mathcal{P}}, \Theta^{\mathcal{P}}=\delta^{*} i^{*} \Omega$, with $i: \kappa^{-1}(\mathcal{P}) \hookrightarrow J^{1} Y^{*}$ and $\Omega$ being the multisymplectic form on $J^{1} Y^{*}$. In the case $Y \rightarrow M$ is endowed with a connection we can proceed as in formula (3.5) and we decompose

$$
\Theta^{\mathcal{P}}=\Theta_{\mathcal{A}}^{\mathcal{P}}+\mathcal{H}
$$

where $\mathcal{H}: \mathcal{P} \rightarrow \bigwedge^{n} T^{*} M$ is the Hamiltonian density.

If the Hamiltonian system is defined by an almost regular Lagrangian density, we have the following equivalence (see [7,13, Section 4.5]).

Proposition 7.2. A section s of the bundle $Y \rightarrow M$ is a critical section of the variational problem defined by $\mathcal{L}$ if and only if $\widehat{\mathbb{F L}} \circ J^{1} s$ is a solution of the Hamiltonian system with constraints.

Hamilton-Cartan equations for electromagnetism. It is straightforward to see that for electromagnetism, $\mathcal{L}$ is not hyper-regular, that is, the Legendre transformation $\widehat{\mathbb{F L}}$ is not a diffeomorphism. Nevertheless, the electromagnetic Lagrangian density is almost regular in the sense given above.

For $Y=T^{*} M$ and the Lagrangian $\mathcal{L}\left(j_{x}^{1} \omega\right)=(1 / 2)\langle\mathrm{d} \omega, \mathrm{d} \omega\rangle_{g} \mathbf{v}_{g}$, the constraint $\mathcal{P}$ defined on $\Pi=T M \otimes T M \otimes \bigwedge^{n} T^{*} M$ is

$$
\mathcal{P}=\widehat{\mathbb{F} \mathcal{L}}\left(J^{1}\left(T^{*} M\right)\right)=(T M \wedge T M) \otimes \bigwedge^{n} T^{*} M \subset \Pi .
$$

Indeed, using the standard coordinates $\left(x^{\mu}, A_{\nu}, \pi^{\mu \nu}\right)$ on $\Pi=(T M \otimes T M) \otimes \bigwedge^{n} T^{*} M$, we have

$$
\pi^{\mu \nu}=\frac{\partial \mathcal{L}}{\partial A_{\mu, v}}=A_{\mu, v}-A_{\nu, \mu},
$$

and then

$$
\pi^{\mu \nu}+\pi^{\nu \mu}=0
$$

We define a change of coordinates on $\Pi$ as

$$
F^{\mu \nu}=\frac{1}{2}\left(\pi^{\mu \nu}-\pi^{v \mu}\right) \quad \text { for } \mu<v, \quad S^{\mu \nu}=\frac{1}{2}\left(\pi^{\mu \nu}+\pi^{v \mu}\right) \quad \text { for } \mu \geq v .
$$

The constraint $\mathcal{P}$ is defined by $S^{\mu \nu}=0$, for all $\mu \geq v$. 
The pull-back of the multisymplectic form $\Omega$ to $\kappa^{-1}(\mathcal{P})$ is locally

$$
\Omega^{\mathcal{P}}=-\mathrm{d} p \wedge \mathbf{v}+\sum_{\mu<v} \mathrm{~d} F^{\mu \nu} \wedge\left(\mathrm{d} A_{\mu} \wedge \mathbf{v}_{v}-\mathrm{d} A_{\nu} \wedge \mathbf{v}_{\mu}\right)
$$

and Eq. (7.2) gives

$$
-\frac{\partial H}{\partial F^{\mu \nu}}+\frac{\partial A_{\mu}}{\partial x^{\nu}}-\frac{\partial A_{v}}{\partial x^{\mu}}=0, \quad \frac{\partial H}{\partial A_{\mu}}+\frac{\partial F^{\mu \nu}}{\partial x^{\nu}}=0,
$$

which are the Maxwell equations $F_{\mu \nu}=A_{\mu, \nu}-A_{\nu, \mu}$ and $F_{v}^{\mu \nu}=0$. For the last formula (and in the sequel) we define

$$
F^{\mu \nu}=-F^{v \mu}
$$

when $\mu>v$.

Poisson forms on the constraint manifold $\mathcal{P}$. For the constraint manifold $\mathcal{P} \subset \Pi$ as defined in (7.3), we say that an $r$ horizontal form $E$ on $\kappa^{-1}(\mathcal{P})$ is Poisson if there exists an $(n-r)$-multivector field $\chi$ such that

$$
\mathbf{i}_{\chi} \Omega^{\mathcal{P}}=\mathrm{d} E,
$$

where $\Omega^{\mathcal{P}}$ is defined in formula (7.4). We study the case $r=n-1$.

Proposition 7.3. The Poisson $(n-1)$-forms on $\mathcal{P}$ are in coordinates

$$
E=\left(F^{\mu v} X_{v}+G^{\mu}\right) \mathbf{v}_{\mu}
$$

where $X_{v}=X_{v}(x)$, are functions on $M$ and $G^{\mu}=G^{\mu}(x, A)$ functions on $T^{*} M$ satisfying

$$
\frac{\partial G^{\mu}}{\partial A_{\nu}}=\frac{\partial G^{v}}{\partial A_{\mu}}
$$

Proof. In coordinates

$$
E=E^{\mu} \mathbf{v}_{\mu}, \quad \mathrm{d} E=\frac{\partial E^{\mu}}{\partial x^{\mu}} \mathbf{v}+\frac{\partial E^{\mu}}{\partial A_{\nu}} \mathrm{d} A_{\nu} \wedge \mathbf{v}_{\mu}+\sum_{\gamma<\rho} \frac{\partial E^{\mu}}{\partial F^{\gamma \rho}} \mathrm{d} F^{\gamma \rho} \wedge \mathbf{v}_{\mu} .
$$

On the other hand, if we have

$$
X=X \frac{\partial}{\partial p}+X_{v} \frac{\partial}{\partial A_{v}}+\sum_{\mu<v} X^{\mu \nu} \frac{\partial}{\partial F^{\mu \nu}}
$$

from (7.4), the condition (7.5) gives

$$
\frac{\partial E^{\mu}}{\partial x^{\mu}}=-X, \quad X^{\mu \nu}=\frac{\partial E^{v}}{\partial A_{\mu}}=-\frac{\partial E^{\mu}}{\partial A_{\nu}}, \quad \text { for } \mu<v, \quad \frac{\partial E^{\mu}}{\partial F^{\gamma \rho}}=\delta_{\gamma}^{\mu} X_{\rho}-\delta_{\rho}^{\mu} X_{\gamma} .
$$

These conditions are satisfied if and only if the form $E$ is as said in the statement. 
Given two Poisson forms $E$ and $H$ on $\mathcal{P}, \operatorname{deg}(E)=r, \operatorname{deg}(H)=s$, we define their Poisson bracket to be

$$
\{E, H\}=(-1)^{n-r} i_{\chi E} i_{\chi H} \Omega^{\mathcal{P}},
$$

where $\chi_{E}$ and $\chi_{H}$ are the multivector fields associated to $E$ and $H$, respectively. For the special case where $E$ is an $(n-1)$-form and $H$ is a function, we have the following local formula:

$$
\{E, H\}=\sum_{\mu<\nu}\left(\frac{\partial E^{\nu}}{\partial A_{\mu}} \frac{\partial H}{\partial F^{\mu \nu}}-\frac{\partial E^{\mu}}{\partial F^{\nu \mu}} \frac{\partial H}{\partial A_{\nu}}\right) .
$$

Theorem 7.4. A section $F$ of the constraint bundle $\mathcal{P} \rightarrow M$ is a solution of the Hamiltonian system $(\mathcal{P}, \mathcal{A}, \mathcal{H})$ if and only if for every Poisson horizontal $(n-1)$-form $E$ on $\mathcal{P}$ we have

$$
\{E, H\} \mathbf{v}=\mathrm{d}\left(F^{*} E\right)-\mathrm{d}^{\mathrm{h}} E \circ F,
$$

where $\mathrm{d}^{\mathrm{h}}$ is the horizontal differential with respect to the Riemannian connection on the bundle $\Pi=T M \wedge T M \otimes \wedge^{n} T^{*} M \rightarrow M$.

Proof. In coordinate system such that $\mathbf{v}=\mathrm{d} x^{1} \wedge \cdots \wedge \mathrm{d} x^{n}$, we have

$$
\{E, H\} \mathbf{v}=\sum_{\mu<\nu}\left(\frac{\partial E^{\nu}}{\partial A_{\mu}} \frac{\partial H}{\partial F^{\mu \nu}}-\frac{\partial E^{\mu}}{\partial F^{\nu \mu}} \frac{\partial H}{\partial A_{\nu}}\right) \mathbf{v}=\frac{\partial G^{\nu}}{\partial A_{\mu}} F_{\mu \nu} \mathbf{v} .
$$

On the other hand,

$$
\begin{aligned}
\mathrm{d}\left(F^{*} E\right)-\mathrm{d}^{\mathrm{h}} E \circ F= & \left(\frac{\partial E^{\mu}}{\partial x^{\mu}}+\frac{\partial E^{\mu}}{\partial A_{\nu}} \frac{\partial A_{v}}{\partial x^{\mu}}+\frac{\partial E^{\mu}}{\partial F^{\rho \gamma}} \frac{\partial F^{\rho \gamma}}{\partial x^{\mu}}-\frac{\partial E^{\mu}}{\partial x^{\mu}}+\frac{\partial E^{\mu}}{\partial A_{\nu}} \Gamma_{\mu \nu}^{\rho} A_{\rho}\right. \\
& \left.-\frac{\partial E^{\mu}}{\partial F^{\rho \gamma}} \Gamma_{\mu \tau}^{\rho} F^{\tau \gamma}-\frac{\partial E^{\mu}}{\partial F^{\tau \gamma}} \Gamma_{\rho \mu}^{\rho} F^{\tau \gamma}\right) \mathbf{v} \\
= & \left(\frac{\partial G^{\mu}}{\partial A_{\nu}}\left(\frac{\partial A_{\nu}}{\partial x^{\mu}}-\frac{\partial A_{\mu}}{\partial x^{\nu}}\right)+X_{\gamma} \frac{\partial F^{\gamma \mu}}{\partial x^{\mu}}\right) \mathbf{v},
\end{aligned}
$$

where we have used the structure of Poisson forms on $\mathcal{P}$ given in Proposition 3.3. So the equality holds true if and only if the Maxwell equations are satisfied.

Reduction. For the action of the gauge group on $\pi_{M Y}: Y=T^{*} M \rightarrow M$ is by affine translations, the action of the gauge bundle $\mathcal{G}=J^{2}(M, \mathbb{R})$ on $V^{*} Y=\pi_{M Y}^{*} T M \rightarrow T^{*} M$ is purely horizontal, that is, it only acts on $T^{*} M$ as in formula (7.1) and leaves the fiber invariant. Therefore, the fiberwise action of $\mathcal{G}$ on the polysymplectic bundle

$$
\Pi=T M \otimes_{T^{*} M} V^{*} Y \otimes_{T^{*} M} \bigwedge^{n} T^{*} M=T M \otimes_{T^{*} M} T M \otimes_{T^{*} M} \bigwedge^{n} T^{*} M \rightarrow T^{*} M
$$

is trivial along the fibers and only effective on $T^{*} M$. Moreover, we have a first-order action, that is, it is really defined by $J^{1}(M, \mathbb{R})$ in the way that two 2-jets $j_{x}^{2} f, j_{x}^{2} f^{\prime}$ such that 
$j_{x}^{1} f=j_{x}^{1} f^{\prime}$ produce the same action. More precisely,

$$
\left(\frac{\partial}{\partial x^{i}} \otimes \frac{\partial}{\partial x^{j}} \otimes \mathrm{d}^{n} x\right)_{\omega_{x}} \cdot j_{x}^{2} f=\left(\frac{\partial}{\partial x^{i}} \otimes \frac{\partial}{\partial x^{j}} \otimes \mathrm{d}^{n} x\right)_{\omega_{x}} \cdot j_{x}^{1} f .
$$

The quotient of this action is simply

$$
\frac{\Pi}{\mathcal{G}}=T M \otimes T M \otimes \bigwedge^{n} T^{*} M \rightarrow M
$$

and

$$
\frac{\mathcal{P}}{\mathcal{G}}=T M \wedge T M \otimes \bigwedge^{n} T^{*} M \rightarrow M
$$

for the constraint. If we define the coordinate system $\left(x^{\mu}, f^{\mu v}\right), \mu<v$, on $\mathcal{P} / \mathcal{G}$, the projection simply reads $\left(x^{\mu}, A_{\mu}, F^{\mu \nu}\right) \mapsto\left(x^{\mu}, F^{\mu \nu}\right)$, that is, $f^{\mu \nu}=F^{\mu \nu}$. It means that functions and forms on $\Pi$ invariant under the gauge group must not depend on the coordinates $A_{\mu}$ when expressed in locally. Then we have the following proposition.

Proposition 7.5. The projection to $\Pi / \mathcal{G}$ of the set of Poisson horizontal $(n-1)$-forms on $\Pi$ invariant under the action of the gauge group is

$$
\left\{e=\left(f^{\mu \nu} X_{v}+g^{\mu}\right) \mathbf{v}_{\mu} \mid X_{v}, g^{\mu} \in C^{\infty}(M)\right\} .
$$

Theorem 7.6. Let $E$ be a Poisson form on $\mathcal{P}$ and $H$ an invariant function, both invariant under the action of the gauge group. Then $\{E, H\}$ identically vanishes.

Proof. Using the local expression (7.6) of $\{E, F\}$, if $\partial E^{\mu} / \partial A_{v}=0, \partial H / \partial A_{v}=0$, we have $\{E, H\}=0$.

Hence the version of Theorem 5.2 for this setting turns out to be very easy.

Theorem 7.7. For the Maxwell Hamiltonian system $\mathcal{P}$, given a section $F$ of the bundle $\Pi \rightarrow M$ the following points are equivalent:

1. F is a solution of the Maxwell equations,

2. for any Poisson form $E$ on $\mathcal{P}$ we have

$$
\{E, H\} \mathbf{v}=\mathrm{d}\left(F^{*} E\right)-\mathrm{d}^{\mathrm{h}} E \circ F,
$$

3. for any Poisson form on the reduced bundle $\Pi / \mathcal{G}$, the reduced section satisfies

$$
0=\mathrm{d}\left(f^{*} e\right)-\mathrm{d}^{\mathrm{h}} e \circ f
$$

4. the reduced section $f$ satisfies the equations

$$
\frac{\partial f^{\mu \nu}}{\partial x^{\nu}}=0
$$


Proof. $1 \Leftrightarrow 2$ is just Theorem 5.2. From Theorem 6.3 we have the $2 \Leftrightarrow 3$ for the left-hand side of the formulas. The right-hand side, is evident if we put $E=\kappa^{*} e, H=\kappa^{*} h, \kappa$ being the projection $\Pi \rightarrow \Pi / \mathcal{G}$.

Finally, for $3 \Leftrightarrow 4$, we have

$$
\mathrm{d}\left(f^{*} e\right)-\mathrm{d}^{\mathrm{h}} e \circ f=\frac{\partial e^{\mu}}{\partial x^{\mu}}+\frac{\partial e^{\mu}}{\partial f^{\beta \gamma}} \frac{\partial f^{\beta \gamma}}{\partial x^{\mu}}-\frac{\partial e^{\mu}}{\partial x^{\mu}}-\frac{\partial e^{\mu}}{\partial f^{\beta \gamma}} \Gamma_{\mu \tau}^{\beta} f^{\tau \gamma}=X_{\gamma} \frac{\partial f^{\mu \gamma}}{\partial x^{\mu}}
$$

for any family $X_{\gamma} \in C^{\infty}(M)$. For $4 \Leftrightarrow 1$, note that the reduced equation (7.7) is the half of the Maxwell equations. The other half is just the reconstruction equation.

\section{Conclusions and future directions}

In this paper we have contributed to both covariant Lagrangian reduction theory and to covariant Poisson reduction theory. One of the reasons that we did not pursue covariant multisymplectic reduction theory is the simple fact that it is well-known that setting the multimomentum map equal to a constant is not appropriate (e.g., for electromagnetism, this would not correspond to constraints) and it is not understood at this time what the covariant analog of this should be.

We developed the theory of covariant Poisson structures a little further and showed that there is a covariant version of Lie-Poisson theory that is parallel to the known covariant Euler-Poincare theory and we also showed that both the covariant Lagrangian reduction and covariant Poisson reduction methodologies work for the case of electromagnetism.

Of course there is much to do still, but we hope that the present paper is a useful contribution towards the goal of developing covariant reduction theory. The main missing ingredient is, as we have indicated, a reduction theory in the multisymplectic context. In addition, many more examples need to be worked out, such as relativistic fluids and plasmas and Yang-Mills fields. Of course there is also the big prize: general relativity.

\section{Acknowledgements}

We thank Pedro Luis García, Jeffrey Lawson, Tudor Ratiu, Steve Shkoller and Jedrez Śniatycki for helpful remarks. The research of JEM was partially supported by NSF grant DMS-0204474 and a Max Planck research award, and MCL was partially supported by DGESIC (Spain) under grant no. BMF2000-1314 and Junta Castilla-Léon (Spain) under grant no. SA085-01.

\section{References}

[1] E. Binz, J. Śniatycki, H. Fischer, Geometry of classical fields, in: North-Holland Mathematics Studies, vol. 154, North-Holland, Amsterdam, 1988.

[2] M. Castrillón López, P.L. García Pérez, T.S. Ratiu, Euler-Poincaré reduction on principal bundles, Lett. Math. Phys. 58 (2001) 167-180. 
[3] M. Castrillón López, J.M. Masqué, The geometry of the bundle of connections, Math. Z. 236 (2001) 797-811.

[4] M. Castrillón López, T.S. Ratiu, S. Shkoller, Reduction in principal fiber bundles: covariant Euler-Poincaré equations, Proc. Am. Math. Soc. 128 (2000) 2155-2164.

[5] H. Cendra, D.D. Holm, M.J.W. Hoyle, J.E. Marsden, The Maxwell-Vlasov equations in Euler-Poincaré form, J. Math. Phys. 39 (1998) 3138-3157.

[6] H. Cendra, J.E. Marsden, T.S. Ratiu, Lagrangian reduction by stages, in: Memoirs, vol. 152, American Mathematical Society, Providence, RI, 2001.

[7] A. Echeverria-Enríquez, M.C. Muñoz-Lecanda, N. Román-Roy, Geometry of multisymplectic Hamiltonian first-order field theories, J. Math. Phys. 41 (2000) 7402-7444.

[8] A. Fernández, P.L. García, C. Rodrigo, Lagrangian reduction and constrained variational calculus, in: Proceedings of the IX Fall Workshop on Geometry and Physics, vol. 4, RSME, 2000, pp. 53-64.

[9] M. Forger, H. Römer, A Poisson bracket on multisymplectic phase space, Rep. Math. Phys. 48 (2001) 211218.

[10] P.L. García, The Poincaré-Cartan invariant in the calculus of variations, in: Proceedings of the Symposia on Mathematica, vol. XIV, Convegno di Geometria Simplettica e Fisica Matematica, INDAM, Rome, 1973, No. 53 \#10037, Academic Press, London, 1974, pp. 219-246.

[11] P.L. García, Gauge algebras, curvature and symplectic structure, J. Diff. Geom. 12 (1977) 209-227.

[12] G. Giachetta, L. Mangiarotti, G. Sardanashvily, New Lagrangian and Hamiltonian Methods in Field Theory, World Scientific, Singapore, 1997.

[13] M. Gotay, J. Isenberg, J.E. Marsden, R. Montgomery, Momentum Maps and the Hamiltonian Structure of Classical Relativistic Field Theories, 1985. http://www.cds.caltech.edu/ marsden.

[14] H. Goldschmidt, S. Sternberg, The Hamilton-Cartan formalism in the calculus of variations, Ann. Inst. Fourier (Grenoble) 23 (1973) 203-267.

[15] C. Günther, The polysymplectic Hamiltonian formalism in field theory and calculus of variations. I. The local case, J. Diff. Geom. 25 (1987) 23-53.

[16] D.D. Holm, B.A. Kupershmidt, The analogy between spin glasses and Yang-Mills fluids, J. Math. Phys. 29 (1988) 21-30.

[17] D.D. Holm, J.E. Marsden, T.S. Ratiu, The Euler-Poincaré equations and semidirect products with applications to continuum theories, Adv. Math. 137 (1998) 1-81.

[18] I.V. Kanatchikov, Canonical structure of classical field theory in the polymomentum phase space, Rep. Math. Phys. 41 (1998) 49-90.

[19] J.E. Marsden, R. Montgomery, P.J. Morrison, W.B. Thompson, Covariant Poisson brackets for classical fields, Ann. Phys. 169 (1986) 29-48.

[20] J.E. Marsden, R. Montgomery, T.S. Ratiu, Reduction, symmetry and phases in mechanics, in: Memoirs of the AMS, vol. 436, American Mathematical Society, Providence, RI, 1990.

[21] J.E. Marsden, T.S. Ratiu, Introduction to Mechanics and Symmetry, Texts in Applied Mathematics, vol. 17, 2nd ed., 1994, Springer, Berlin, 1999.

[22] J.E. Marsden, J. Scheurle, The reduced Euler-Lagrange equations, Fields Inst. Commun. 1 (1993) $139-164$.

[23] J.E. Marsden, S. Shkoller, Multisymplectic geometry, covariant Hamiltonians and water waves, Math. Proc. Camb. Philos. Soc. 125 (1999) 553-575.

[24] J.E. Marsden, A. Weinstein, The Hamiltonian structure of the Maxwell-Vlasov equations, Physica D 4 (1982) 394-406.

[25] J.E. Marsden, A. Weinstein, Coadjoint orbits, vortices and Clebsch variables for incompressible fluids, Physica D 7 (1983) 305-323.

[26] J.E. Marsden, A. Weinstein, Comments on the history, theory, and applications of symplectic reduction, in: N. Landsman, M. Pflaum, M. Schlichenmaier (Eds.), Quantization of Singular Symplectic Quotients, Birkhäuser, Boston, 2001, pp. 1-20.

[27] G. Sardanashvily, Generalized Hamiltonian formalism for field theory, World Scientific, River Edge, NJ, 1995.

[28] D.J. Saunders, The geometry of jet bundles, in: London Mathematical Society Lecture Notes Series, vol. 142, Cambridge University Press, Cambridge, 1989.

[29] J. Śniatycki, Regularity of constraints and reduction in the Minkowski space Yang-Mills-Dirac theory, Ann. Inst. H. Poincaré Phys. Théoret. 70 (1999) 277-293. 